\title{
اقتصاديات إنتاج وتسويق محصول الياسمين المصري
}

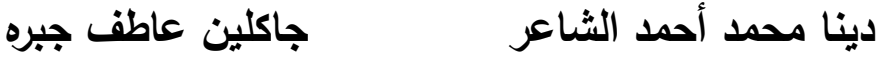

محمد إبراهيم محمد الشهاوي

قسم الاقتصاد الزراعي - كلية الزراعة (سابا بانشا) - جامعة الاسكندرية

الملخص: استهدف البحث دراسة اقتصاديات إنتاج وتسويق محصول الياسمين المصري خلال الفترة (.... ب-

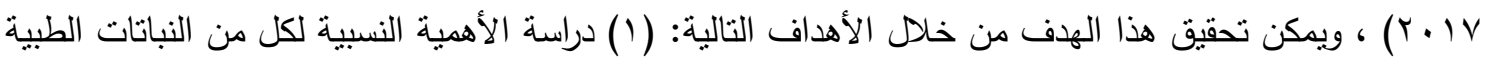
والعطرية والياسمين بالنسبة للمساحة المنزرعة، (r) دراسة تطور بعض المتغيرات الاقتصادية الهامة المرتبطة باقتصاديات إنتاج وتسويق النباتات الطبية والعطرية ومحصول الياسمين والتتبؤ المستقبلي بهم ، (r) تقييم للوضع التصديري للنباتات الطبية والعطرية ومنتجات الياسمين (ع) التوصل إلى مجموعة من التوصيات التي يمكن أن تقيد واضعي السياسة الاقتصادية في هذا المجال.

وقد أوضحت نتائج البحث ما يلي: (1) تمثل الأهمية النسبية لمحصول الياسمين حوالي 00. • \% من

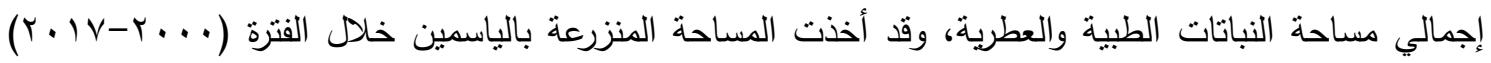

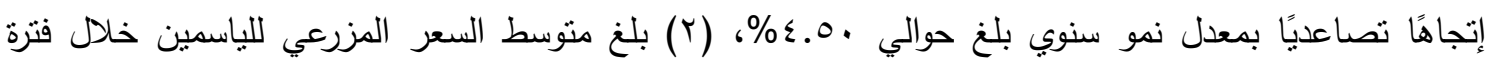

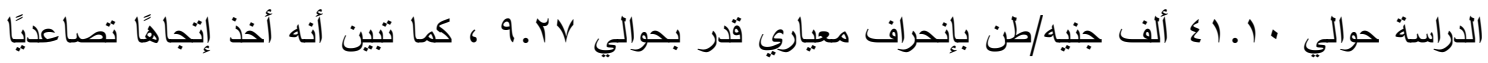

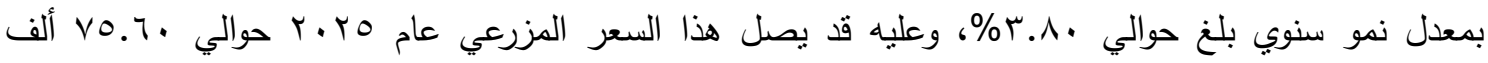

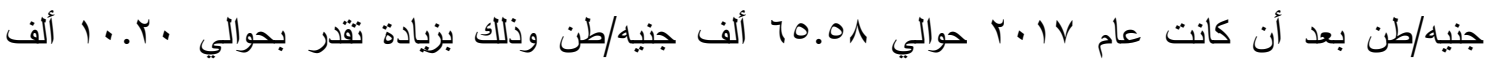

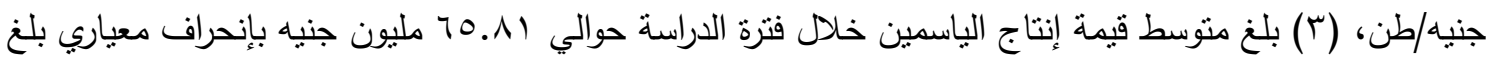

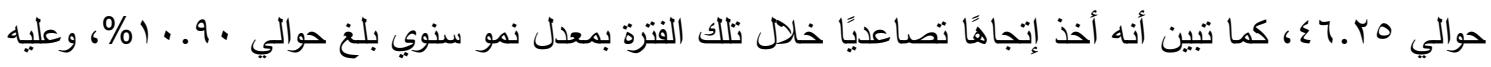

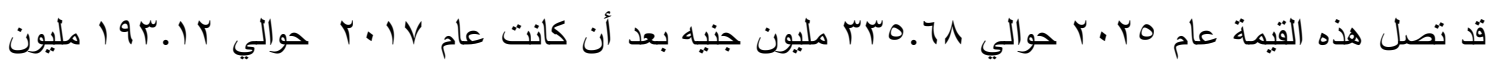

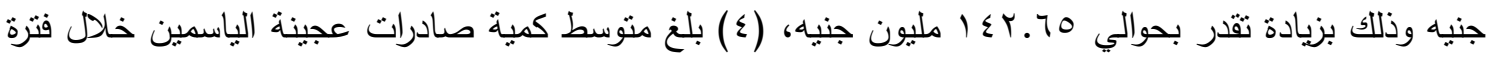

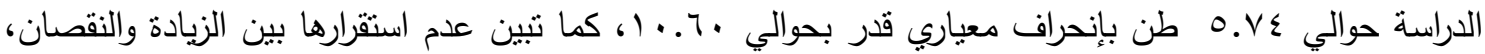
وقدر مقدار التغير عند المتوسط حوالي سبی.. . طن، (0) بلغ متوسط قيمة صادرات عجينة الياسمين خلال فترة

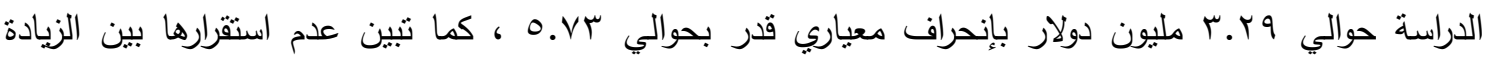

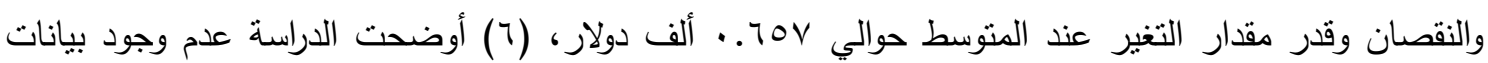
كافية ووافية من صادرات زيت الياسمين العطري مما يدل علي ضعف قاعدة البيانات المتوفرة عن منتجات محصول الياسمين وبصفة خاصة زيت الياسمين، (V) يأخذ المسلك التسويقي لمحصول الياسمين في التسويق الداخلي مسارين هما: المسار الأول: يقوم المزارع بجمع محصوله من أزهار الياسمين ونقله مباشرة للمصنع ويتم عملية البيع والثراء، والمسار الثاني: يقوم المزارع بجمع المحصول من أزهار الياسمين وبيعه إلى الوسيط (المجمع) ثم يقوم المجمع بنقل وبيع المحصول للمصنع مباشرة و (^) يعتبر محصول الياسمين من المحاصيل التصديرية

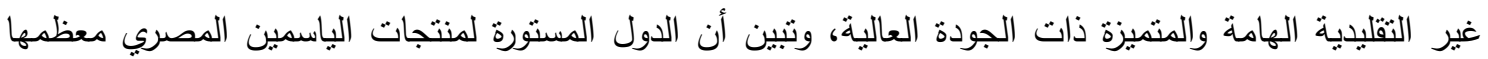

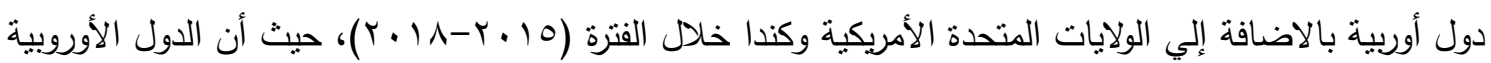
تفضل عجينة وزيت الياسمين المصري عن منافسه الهندي. وتوصي الدراسة في ضوء النتائج المتحصل عليها بما يلي: (1) يجب نوني نوفير البيانات عن الأسواق الداخلية والخارجية والخدمات التسويقية للباسمين من نقل وتخزين وتسويق وغيرها، (ץ) التغلب على المعوقات التى 
تواجه محصول الياسمين سواء أثتاء الانتاج والتسويق، (ب) العمل على تدعيم تتافسية منتجات الياسمين للسوق

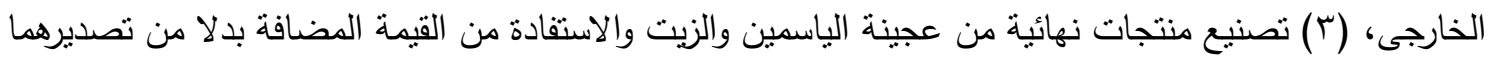
خام، (ع) توفير مصادر تمويل مناسبة من حيث طريقة التعامل وسعر الفائدة والوقت الذي يتتاسب مع المنتجين الحاليين والمرتقبين، (0) تشجيع تكوين تعاونيات إنتاجية وتسويقية مرتبطة بمزارعي الياسمين علي مستوي

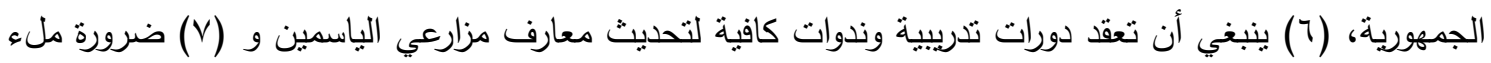

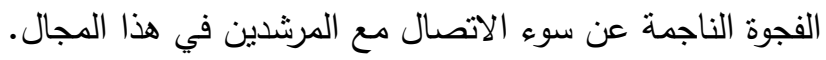
الكلمات الدالة: الانتاج ، التسويق ، الصادرات، الايرادات، المسلك التسويقي، التتبؤ ، الياسمين، النباتات الطبية والعطرية.

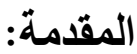

تعتبر النباتات الطبية والعطرية بصفة عامة والياسمين بصفة خاصة من الحاصلات الزراعية غير التقليدية الهامة

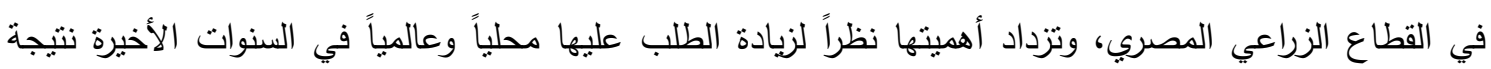
لإستخدامها في صناعة الأدوية، كما أنها تدخل في صناعة العطور ومستحضرات التجميل ومكسبات الطعم التي

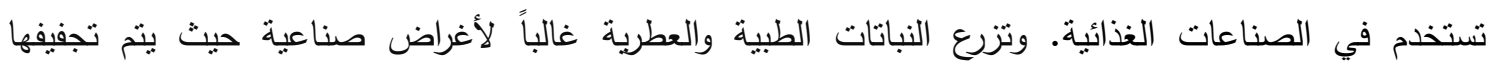
لإستخراج الزيوت العطرية منها، وقد بلغت المساحة المنزرعة في مصر من النباتات الطبية والعطرية حوالي 91.0V

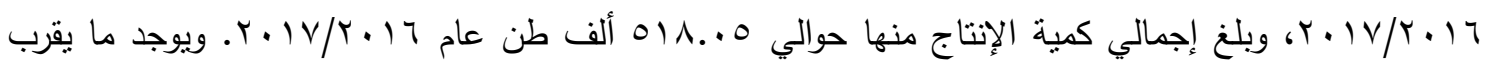
من ألفي نوع من النباتات الطبية والعطرية منها ما ينمو برياً في وادي النيل وفي الصحراء الثرقية والغربية، ومنها ما يزرع للأغراض الصناعية والتي تستخدم إما للتجفيف أو لإستخلاص الزيوت العطرية ولنية ومن أهمها: البابونج،

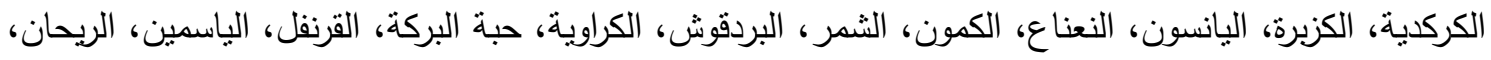

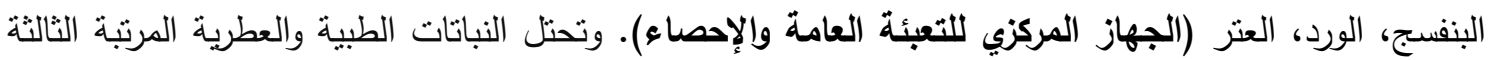

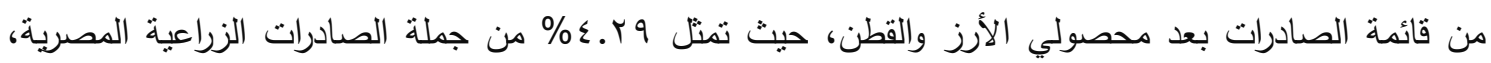
والتي بلغت قرابة بر. .ـ191 مليون دولار المشكلة البحثية: تتبنى السياسة الزراعية المصرية تتمية الصادرات الزراعية وذلك من خلال العمل على تتويعها والتوسع

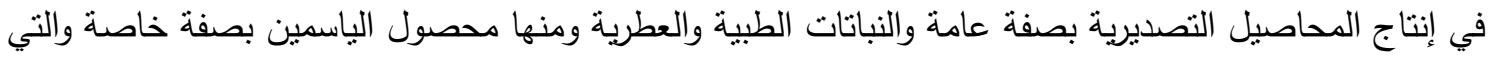
تتمتع بميزة تتافسية في تصديرها، وقد أوضحت الدراسات أن الكثير من النباتات الطبية والعطرية يصعب زراعنها في بعض المناطق في أوروبا وأمريكا خاصة في فصل الشتاء، في حين تتوافر مقومات إنتاجها في مصر حيث الموقع الجغرافي والمناخ المعتدل الملائم لإنتاج تللك النباتات، وعلى الرغم من أهمية الدور التي يمكن أن تؤديه

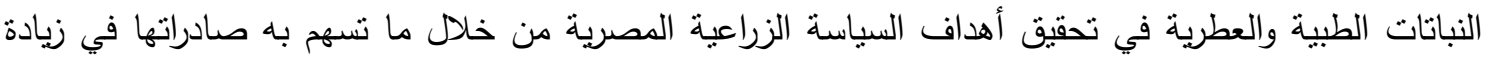
حصيلة الدولة من النقد الأجنبي، ومن ثم تحسين الميزان التجاري الزراعي للدولة، وعلى الرغم من الإهتمام

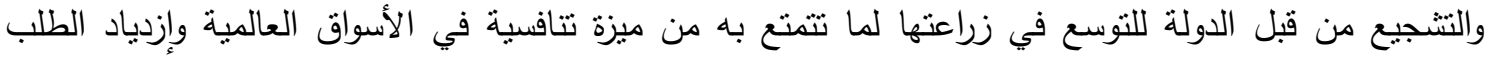

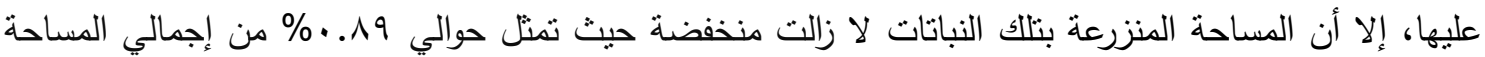

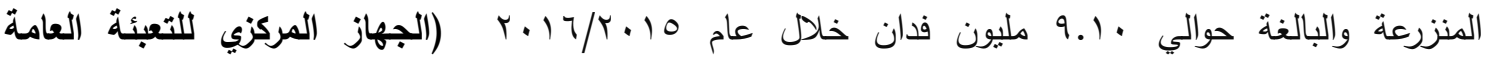


الأمر الذي ييرز أهمية دراسة إنتاج وتسويق الياسمين كأحد أهم النباتات الطبية والعطرية الهامة في

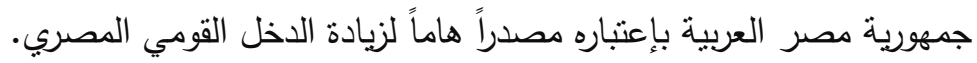

\section{الأهداف البحثية:}

تستهدف الدراسة التعرف على كل من الكفاءة الإنتاجية والتسويقية لمحصول الياسمين في جمهورية مصر العربية، ويمكن تحقيق ذلك من خلال تحقيق الأهداف التالية: (1) دراسة الأهمية النسبية لكل من النباتات الطبية والعطرية والياسمين بالنسبة للمساحة المنزرعة، (r) دراسة نظور بعض المتغيرات الاقتصادية الهامة المرنبطة باقتصاديات إنتاج وتنويق النبانات الطبية والعطرية ومحصول الباسمين والتتبؤ المستقبلي بهم، (r) تقييم للوضع التصديري للنباتات الطبية والعطرية ومنتجات الباسمين و (ع) التوصل لمجموعة من التوصيات النابعة من النتائج المتحصل عليها التي يمكن أن تقيد واضعي السياسة الاقتصادية المصرية في هذا المجال.

مصادر البيانات:

تعتمد الدراسة في تحقيق أهدافها على البيانات الثانوية المنشورة وغير المنشورة والتي نم الحصول عليها من الجهات المختلفة الحكومية وغير الحكومية، متل الجهاز المركزي للتعبئة العامة والإحصاء، وزارة الزراعة وإستصلاح الأراضي، منظمة الأغذية والزراعة (الفاو) وذلك إلى جانب البحوث والمؤتمرات والرسائل العلمية التي إهتمت بموضوع الدراسة وبعض المواقع على شبكة الإنترنت.

\section{الأسلوب البحثي والتحليلي:}

لتحقيق الأهداف المنشودة من الدراسة إعتمدت الدراسة على أسلوب التحليل الكمي والوصفي والمنمثلة فى كل من الجداول التكرارية والنسب المئوية والمتوسطات، نماذج الإنحدار البسيط فى الصور الخطية والتربيعية والنصف لوغاريتمية واللوغاريتمية المزدوجة للمتغيرات الإقتصادية موضع الدراسة وإختيار أفضلها وفقاً للمنطق الإقتصادى والإحصائى والرياضي، فضلا عن نموذج الأريما في التتبؤ ، ومعادلة النمو .

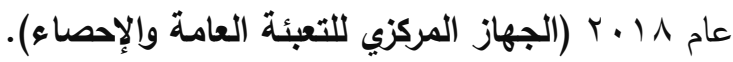

\section{النتائج البحثية ومناقثتها:}

1 ا الأهمية النسبية لمساحة النباتات الطبية والعطرية:

يتبين من الجدول رقم (1) أن متوسط المساحة المنزرعة بالنباتات الطبية والعطرية في مصر خلال الفترة

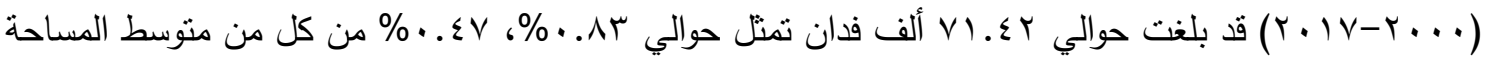
المنزرعة والمساحة المحصولية بفس الترتيب، والتي بلغت في متوسطها حوالي 1.07 ، 10.1 مليون فدان،

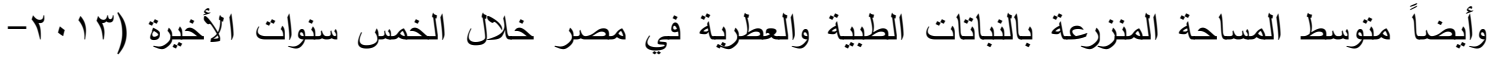

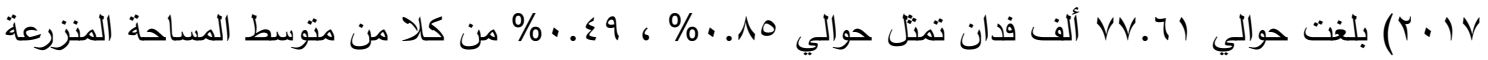

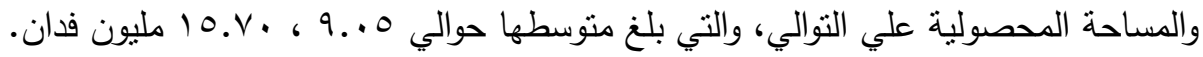


r ا بأهمية النسبية لمساحة محصول الياسمين:

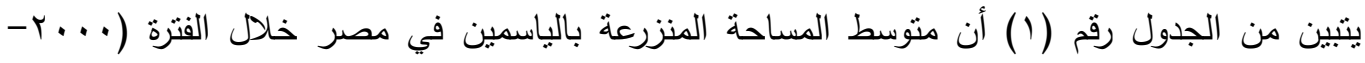

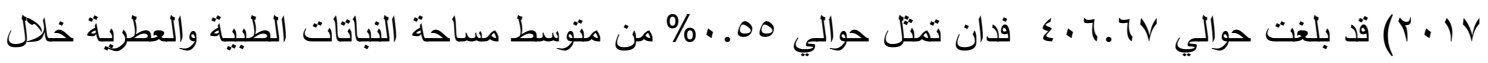

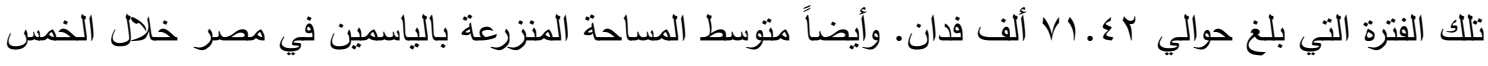

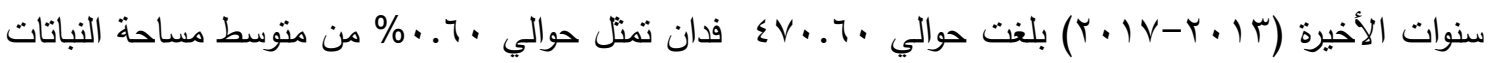

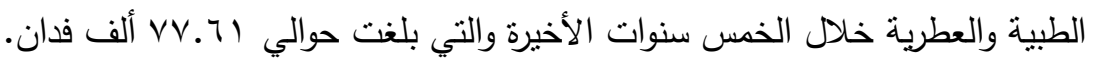

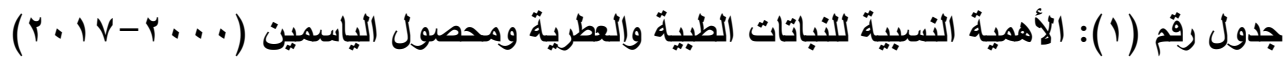

\begin{tabular}{|c|c|c|c|c|c|c|c|}
\hline النباتات الطمين من & (بالياسمين & المحصولية & المنزرعة & المحصولية & المنزلماحة & والطباتباتة & السنوات \\
\hline 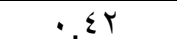 & Tr. &.$\Gamma \Lambda$ &. $.7 \Lambda$ & $1 T .95$ & $V . V Y$ & OY.YT & r... \\
\hline$\therefore \Lambda$ & rIT & •. $\leqslant$. & $\cdot . v \cdot$ & $1 \varepsilon . . r$ & $\vee .90$ & $00 . \Sigma \Lambda$ & $r \ldots l$ \\
\hline תT. & $19 \mathrm{~V}$ & . &. .17 & $1 \leqslant . r_{0}$ & 1.10 & $7 . \wedge \varepsilon$ & $r \ldots r$ \\
\hline . ז人 & . & $\cdot \varepsilon r$ & $\therefore$ Vo & $1 \leqslant . \leqslant V$ & 1.11 & 7.90 & $r \ldots r$ \\
\hline .0 . & rot & $\therefore \leqslant 9$ &.$\wedge \mathrm{V}$ & $1 \leqslant .00$ & $\Lambda . Y \Lambda$ & VI.人T & $r \ldots \varepsilon$ \\
\hline $1 . \cdot \varepsilon$ & VIT & $\cdot \leqslant 7$ & •.AT & $1 \leqslant .9$ & $\Lambda . r \Lambda$ & 71.90 & $r \ldots o$ \\
\hline$\therefore V Y$ & $\{07$ & $\cdot \varepsilon r$ & $\because$. vo & $1 \leqslant .94$ & ᄉ. $\varepsilon 1$ & 74.97 & $r \ldots T$ \\
\hline .01 & sor & $.0 Y$ & .94 & 10.11 & 1. $\varepsilon r$ & $\vee \wedge .0$. & $r \ldots V$ \\
\hline$\cdot .71$ & 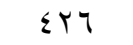 & 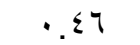 & . & $10 . Y \varepsilon$ & $\Lambda . \varepsilon r$ & 79.90 & $r \ldots \lambda$ \\
\hline. Or & $\{00$ & .07 &. .99 & $10 . \leqslant 9$ & А. $\vee \wedge$ & $\Lambda V_{.} \cdot \varepsilon$ & r...q \\
\hline$\therefore \leqslant \wedge$ & $\varepsilon \cdot r$ & .00 & $.9 \mathrm{~V}$ & $10 . \pi T$ & A. $\vee \varepsilon$ & $\Lambda \varepsilon . \varepsilon$ & $r .1$. \\
\hline . Or & rᄉq & $\cdot . \leqslant V$ & .10 & $10 . r 0$ & 1. Tr & $\forall Y . \wedge \varepsilon$ & $r .11$ \\
\hline • & $\leqslant 0 \leqslant$ & $\therefore \leq 0$ & $\cdot \lambda$ & $10.0 \mathrm{~V}$ & $\wedge . \wedge$. & $V \cdot . \Sigma T$ & T.IT \\
\hline .09 & אדי & $\cdot . \varepsilon$ & .79 & $10 . \leqslant 9$ & 1.90 & 71.57 & $r .1 T$ \\
\hline $.0 \leqslant$ & rᄉr & $\cdot . \leqslant 0$ & $\cdot . \wedge \cdot$ & $10 . \mathrm{V}$ & 1.94 & $v 1.0$ & $r \cdot 1 \leqslant$ \\
\hline$\cdot .71$ & $\{71$ & $\cdot \varepsilon \wedge$ & $\cdot . \Lambda \Gamma$ & $10.7 \varepsilon$ & 9.1. & vo.vo & $r+10$ \\
\hline •. & 017 &. .01 & .199 & 10.1. & 9.1 . & NI.YY & $r .17$ \\
\hline • & וTד & ת & $1 . \cdot v$ & 10.17 & 9.19 & $91.0 \mathrm{~V}$ & $r .1 V$ \\
\hline .00 & $\varepsilon .7 .7 \mathrm{~V}$ & $\cdot . \leqslant V$ & $\cdot . \wedge r$ & 10.1. & 1.07 & $V 1 . \leqslant Y$ & المتوسط العام \\
\hline .7 & $\varepsilon V \cdot .7$. &..$\leqslant 9$ & $\cdot . \wedge 0$ & 10.1. & 9.0 & VV.TI & 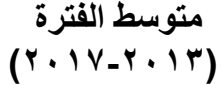 \\
\hline .17 & Irv.qr & $\because 4$ & .11 & $\cdot .71$ & $\because \leqslant Y$ & 11.71 & المعياري \\
\hline
\end{tabular}

المصدر:جمعت وحسبت من بيانات الجهاز المركزي للتعبئة العامة والإحصاء، أعداد متفرقة.

r. بعض المتغيرات الإنتاجية والإقتصادية للنباتات الطبية والعطرية:

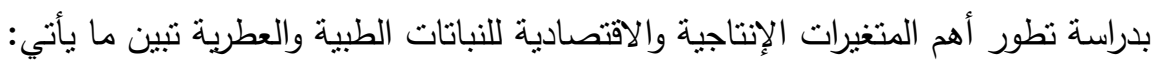

- المساحة المنزرعة بالنباتات الطبية والعطرية:

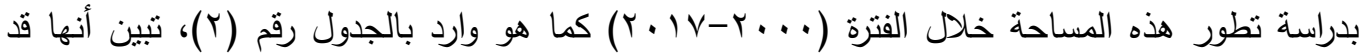

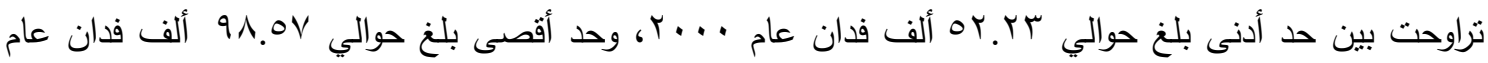




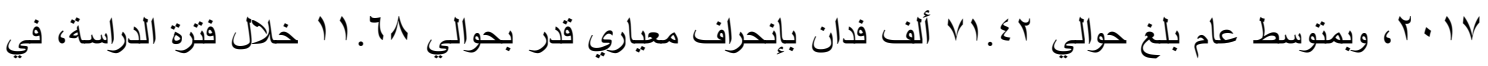

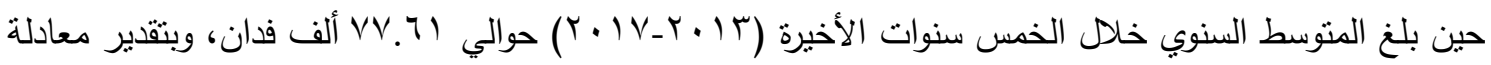

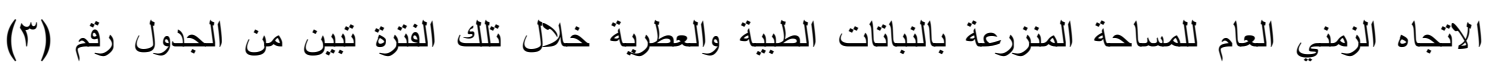

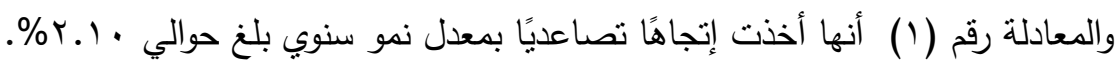

وبدراسة التتبؤ للمساحة المنزرعة بالنباتات الطبية والعطرية كما هو مبين بالجدول رقم (r)، تنين أن

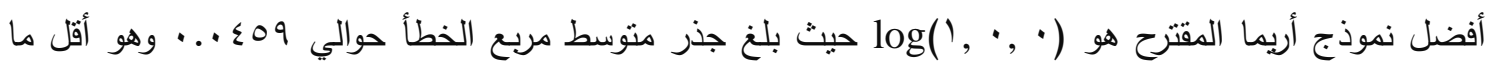

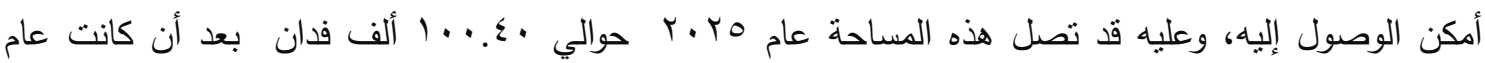

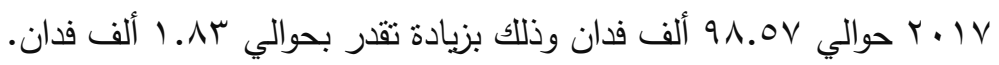

\section{- الإنتاجية الفدانية للنباتات الطبية والعطرية:}

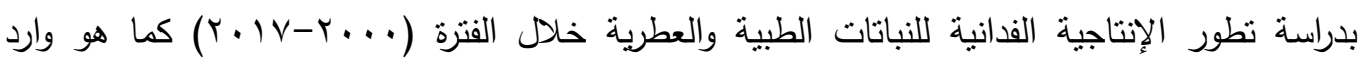

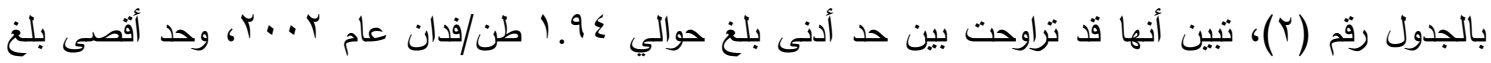

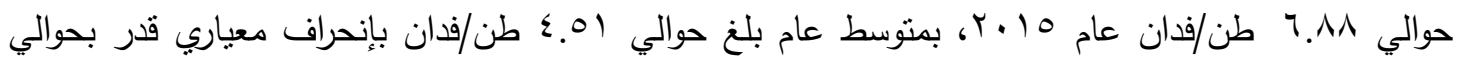

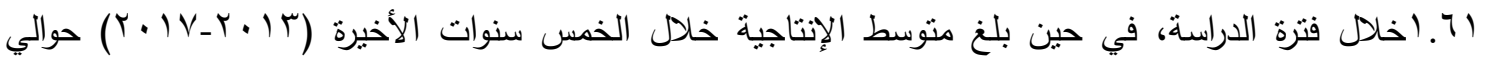

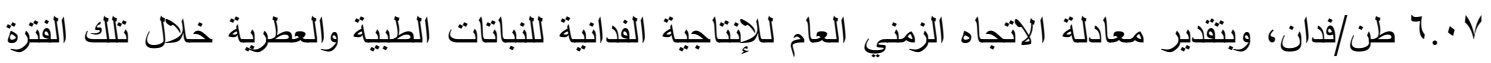

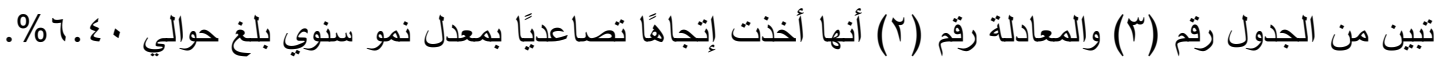
وبدراسة التتبؤ للانتاجية الفدانية للنباتات الطبية والعطرية تبين أن أفضل نموذج أريما مقترح هو ( , . , أ

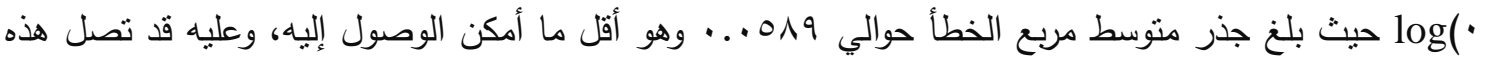

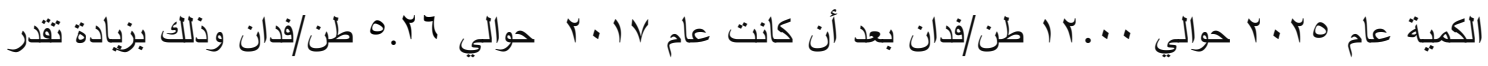

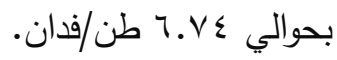

\section{- كمية الإنتاج للنباتات الطبية والعطرية:}

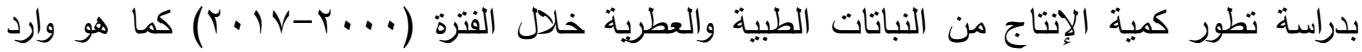

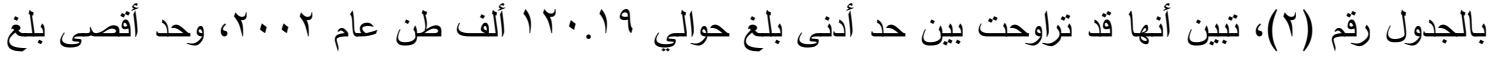

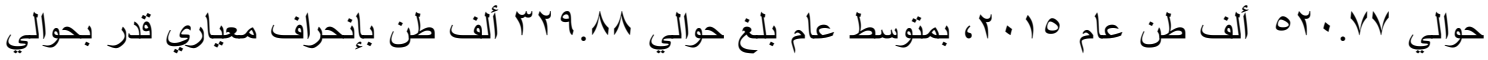

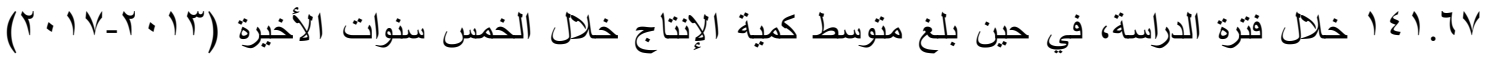
حوالي •9.47 ألف طن، وبتقدير معادلة الاتجاه الزمني العام لهذا المتغير خلال تلك الفترة تبين من الجدول رقم

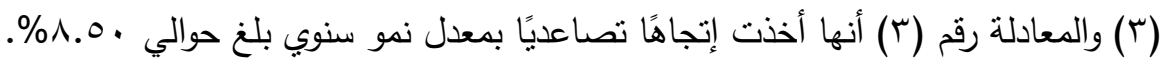
وبدراسة التتبؤ لكمية الإنتاج بالنباتات الطبية والعطرية تبين قد تصل هذه الكمية عام هr.r حوالي

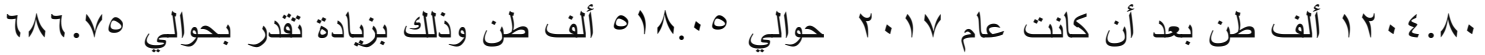
ألف طن.

\section{- قيمة الإنتاج للنباتات الطبية والعطرية:}

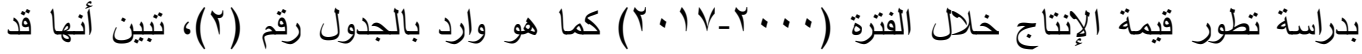

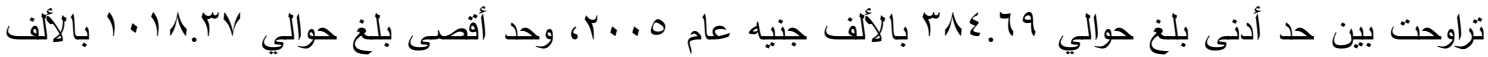




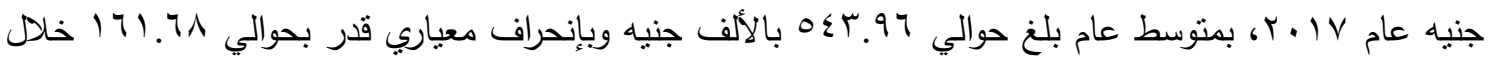

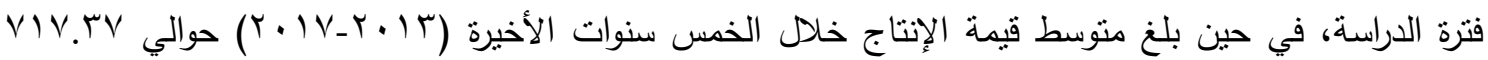
بالألف جنيه، وبتقدير معادلة الاتجاه الزمني العام لقيمة الانتاج من النباتات الطبية والعطرية خلال تلإلك الفترة تنين

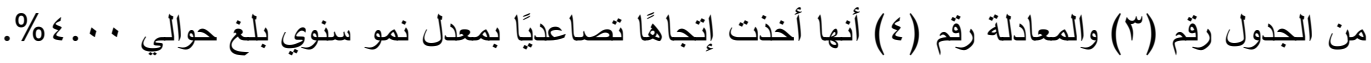
جدول رقم (ץ): الوضع الحالي والمستقبلي لأهم المتغيرات الإنتاجية والاقتصادية المرتبطة بالنباتات الطبية

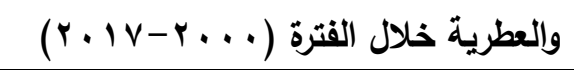

\begin{tabular}{|c|c|c|c|c|}
\hline (بالالفة جنيمة) & كمبة الإنتاج & (طن/فاجانة) & (بالألف فدانة) & السنوات \\
\hline$\varepsilon r 0 . r$ & 10r.s. & r.9r & OY.YT & r... \\
\hline$\sum r 0 . \mu$ & 17.7. & $r .9 \varepsilon$ & 00.21 & $r \ldots l$ \\
\hline גז.TזE & $15 \cdot .19$ & $1.9 \varepsilon$ & $\Pi . \wedge \varepsilon$ & $r \ldots r$ \\
\hline $01 Y . Y 4$ & 197.94 & זr. & 7.90 & $r \ldots r$ \\
\hline$r q \leqslant . \leqslant 7$ & $19 \vee . \wedge \varepsilon$ & r.vo & VI. 14 & $r \ldots \varepsilon$ \\
\hline$r \wedge \varepsilon .79$ & $178 . .$. & $r . \varepsilon r$ & 71.90 & $r \ldots o$ \\
\hline$\varepsilon \leqslant V .9$ & $1 v 0.0$. & r.vq & $7 r .97$ & $r \ldots r$ \\
\hline צr & זr.זרץ & 0.1 & $\vee \wedge .0$. & $r \ldots r$ \\
\hline$\varepsilon 19 . r v$ & & $\leqslant .10$ & 79.90 & $r \ldots \lambda$ \\
\hline 000.10 & $\varepsilon .9 .79$ & $\varepsilon . \vee 1$ & $\Lambda V_{.} \cdot \varepsilon$ & $r \ldots q$ \\
\hline OVq. $\leqslant Y$ & rq9.r & $\varepsilon . V T$ & $\Lambda \leq . \leq$. & $r \cdot 1$. \\
\hline OVY.IV & $\{r \mid . \wedge r$ & $0 . \wedge \Lambda$ & $V Y . \wedge \varepsilon$ & $r .11$ \\
\hline $7 . .14$ & $\sum 77.01$ & אד.7 & $V \cdot . \Sigma r$ & $r .1 T$ \\
\hline$\{91.07$ & . & 0.91 & $71 . \leqslant 7$ & $r .1 T$ \\
\hline 7.7 .71 & $\leqslant 70.0 \leqslant$ & 7.00 & $v 1.00$ & r.l $\varepsilon$ \\
\hline$T \leqslant 1 . \leqslant V$ & or. .rV & $7 . \wedge \wedge$ & VO.VO & $r .10$ \\
\hline AYI.VE & $\sum T V .+Y$ & 0.10 & NI.YY & $r .17$ \\
\hline $1.1 \wedge . r v$ & $011 . .0$ & $0 . T_{T}$ & $91.0 \mathrm{~V}$ & $r .1 V$ \\
\hline 074.11 & $r Y q . \wedge \wedge$ & $\{.01$ & $V 1 . \leqslant Y$ & المتوسط العام \\
\hline Vqv.rv & $\leq 74.9$. & $7 . \cdot V$ & VV.7I & 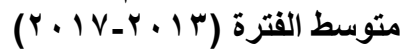 \\
\hline$r r q . \leqslant q$ & $1 \leqslant 1.7 \mathrm{~V}$ & 1.71 & 11.71 & الاتحراف المعياري \\
\hline \multicolumn{5}{|c|}{ تنبؤ الأريما } \\
\hline 970.0. & ᄉт!.11 & 9.r. & 9r.7. & $r \cdot r)$ \\
\hline ११^.५. & $94 \leqslant .07$ & 9.9. & $9 \leq . \leq$. & $r \cdot r r$ \\
\hline 1.40 .7$. & $I \cdot r \cdot V \wedge$ & $1 . .7$ & $97 . \%$. & t.rt \\
\hline $1 . \times 7 .$. & $111 . .199$ & $11 . r$. & q^.५. & $r \cdot r \varepsilon$ \\
\hline$\| 1 \wedge . \wedge$. & $M \cdot \Sigma . \wedge$. & ir... & $1 \cdots \varepsilon$. & r. ro \\
\hline
\end{tabular}

* التتبؤ بإستخدام نماذج الأريما (ARIMA) بطريقة البونستراب (Bootstrap) ** * * مساب الإنتاجية بقسمة الإنتاج على المساحة لفترة التتبؤ. المصدر: جمعت وحسبت من بيانات الجهاز المركزي للتعبئة العامة والإحصاء. Sources: http://www.fao.org/faostat/en/\#data 
جدول رقم (r): معادلات الإتجاه الزمني ونماذج الأريما لتطور المتغيرات الإنتاجية والإتصادية للنباتات الطبية

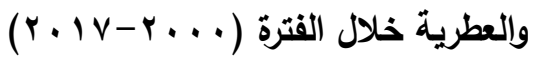

\begin{tabular}{|c|c|c|c|c|c|}
\hline No. & Variables & Equation* & $\mathrm{R}^{2}$ & F & P-Value \\
\hline 1 & 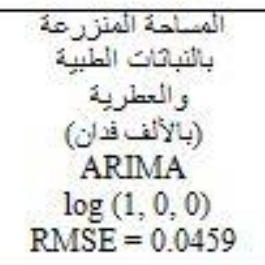 & $\begin{array}{c}\operatorname{Ln} \mathrm{Y}_{1}=57.554+0.021 \mathrm{X} \\
\quad(17.215) \quad(3.989) \\
\log \mathrm{Y}_{1}=0.4212 \log \mathrm{AR}^{* *}(1)\end{array}$ & 0.50 & 15.91 & 0.001 \\
\hline 2 & 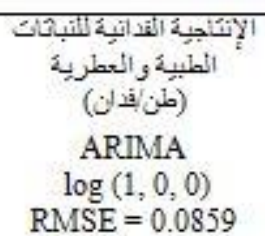 & $\begin{array}{l}\operatorname{LY}_{2}=2.296+0.0641 \mathrm{X} \\
\quad(9.622) \quad(6.639) \\
\log \mathrm{Y}_{2}=0.2874 \log \mathrm{AR}^{* *}(1)\end{array}$ & 0.73 & 44.08 & 0.000 \\
\hline 3 & 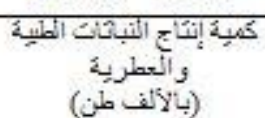 & $\begin{aligned} \operatorname{Ln} Y_{3} & =132.253+0.085 X \\
& (9.391) \quad(8.638)\end{aligned}$ & 0.82 & 74.61 & 0.000 \\
\hline \multirow[t]{2}{*}{4} & 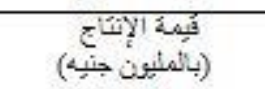 & $\begin{aligned} \operatorname{Ln} Y_{4}= & 360.157+0.040 X \\
(13.323) & (5.736)\end{aligned}$ & 0.67 & 32.90 & 0.000 \\
\hline & $\begin{array}{c}\text { ARIMA } \\
\log (1,1,1) \\
\text { RMSE }=0.0594\end{array}$ & $\begin{array}{l}\mathrm{Y}_{4}=0.48391 \log \mathrm{AR}^{* *}(1)+1.0483 \log \\
\mathrm{MA}^{* * *}(1)\end{array}$ & & & \\
\hline
\end{tabular}

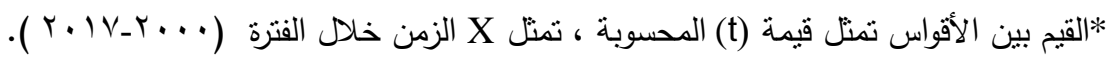
Auto Regressive (AR)** الإنحدار المنسلسل. Mouving Average (MR)**** المنوسط المتحرك. المصدر: جمعت وحسبت من نتائج تحليل الإتجاه الزمني ونماذج الأريما لبيانات الجدول رقم (Y).

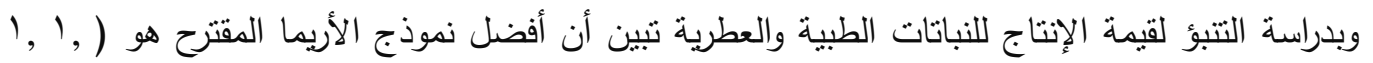

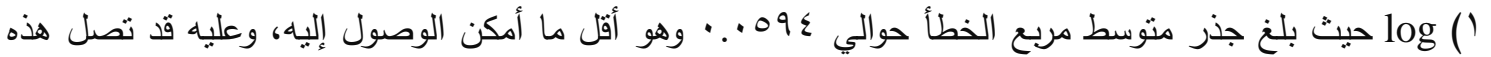

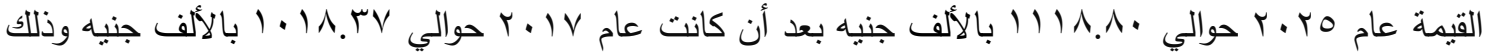
بزيادة تقدر بحوالي سع. • . 1 بالألف جنيه. ؛ ـ بعض المتغيرات الإنتاجية والإقتصادية للياسمين: بدراسة تطور أهم المتغيرات الإنتاجية والاقتصادية للياسمين تنين ما يأني:

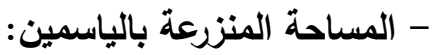

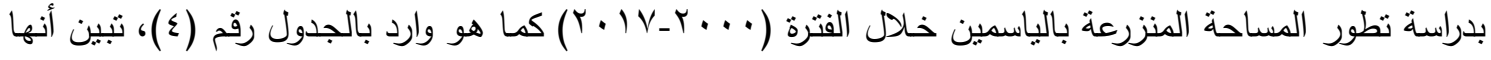

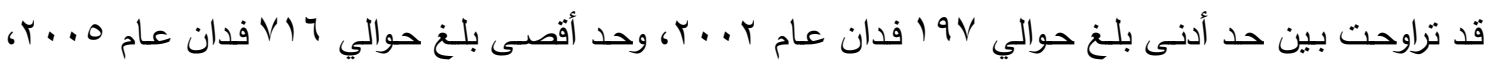

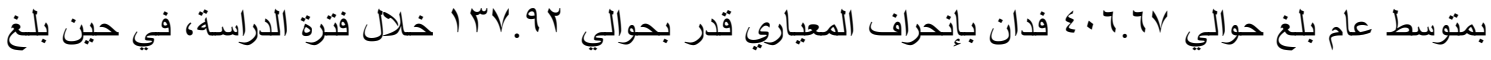
منوسط المساحة المنزرعة 


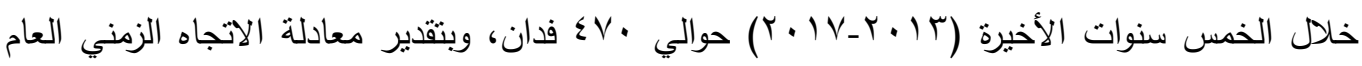

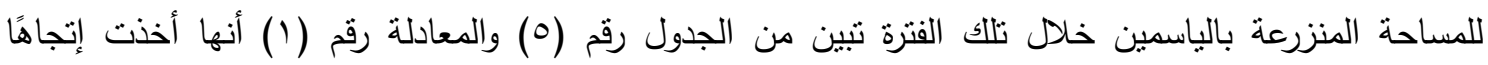

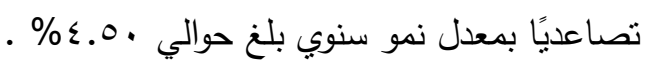

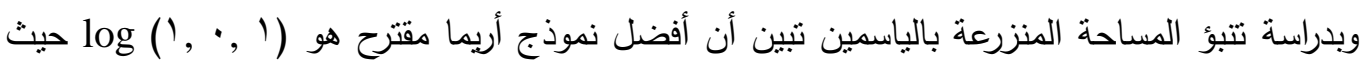

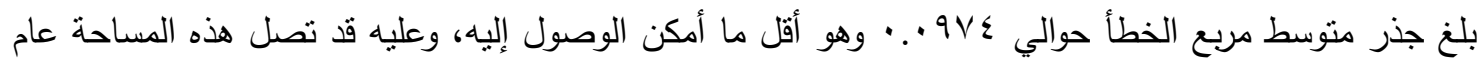

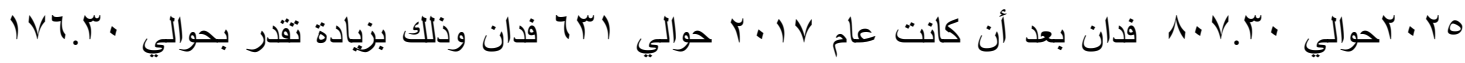

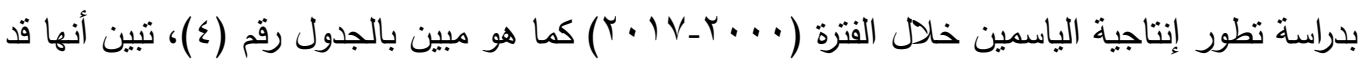

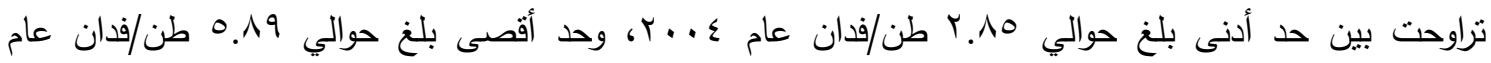

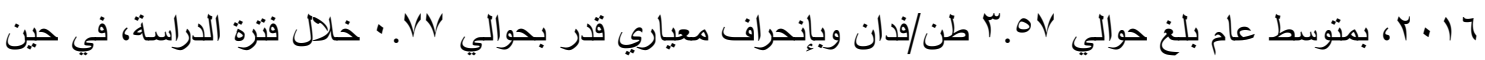

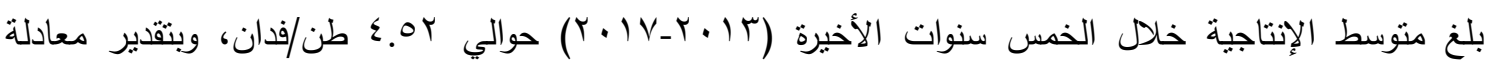
الاتجاه الزمني العام لهذا المتغير خلال تلك الفترة تبين من الجدول رقم (0) والمعادلة رقم (ب) أنها أخذت إتجاهًا

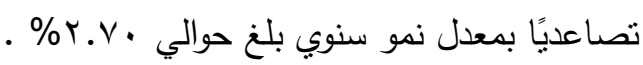

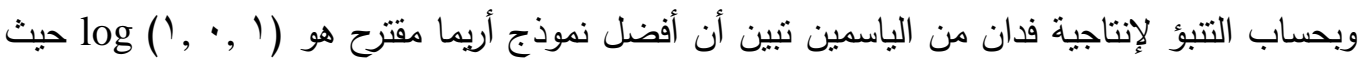
بلغ جذر متوسط مربع الخطأ حوالي إه1..

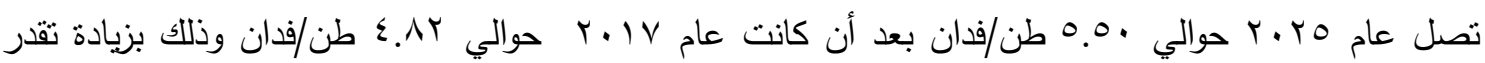

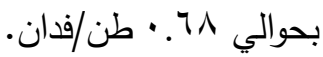

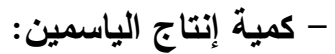

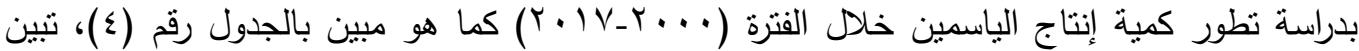

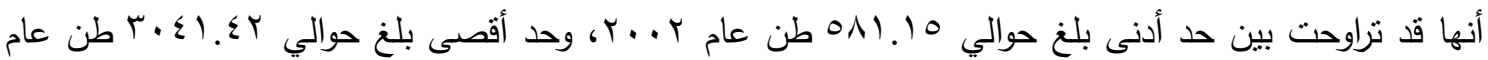

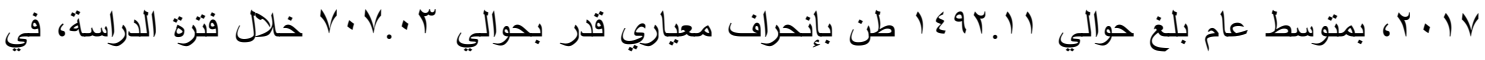

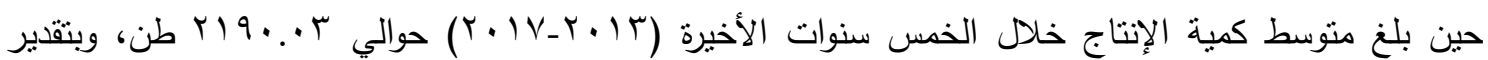
معادلة الاتجاه الزمني العام لكمية إنتاج الياسمين تنين من الجدول رقم (0) والمعادلة رقم (ץ) أنها أخذت إتجاهًا

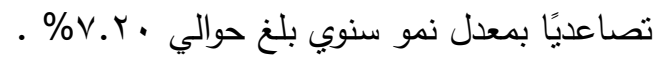

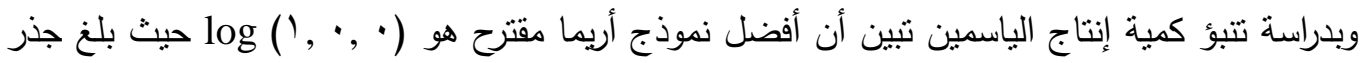

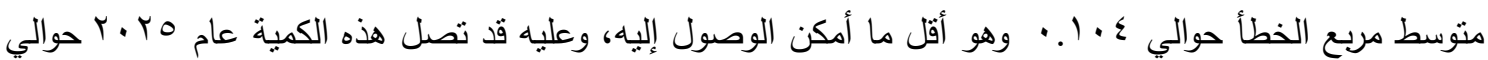

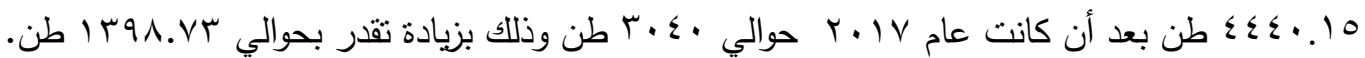

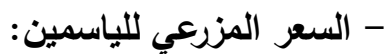

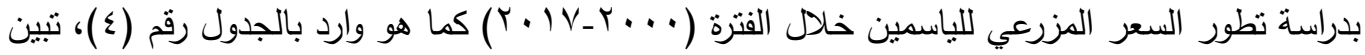

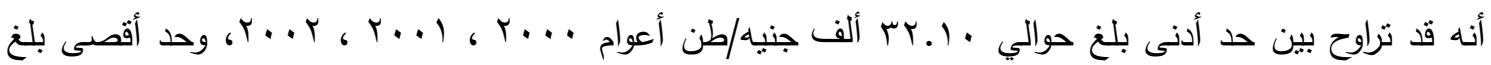

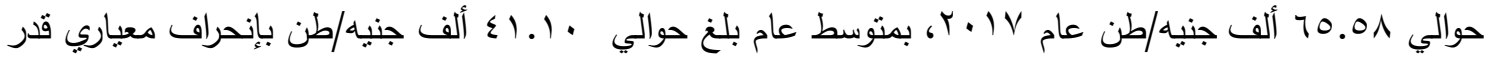
بحوالي V.Y.T خلال فترة الدراسة، في حين بلغ منوسط السعر المزرعي للياسمين خلال الخمس سنوات الأخيرة 


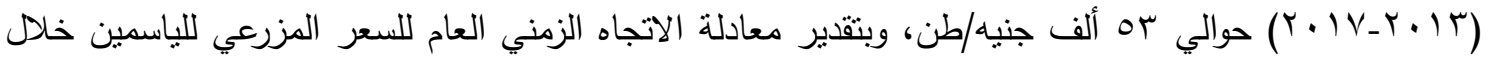

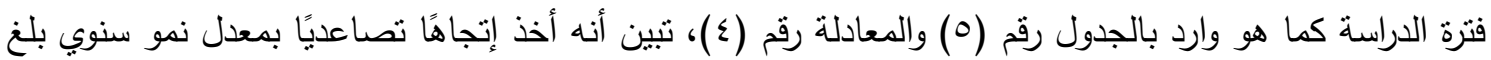

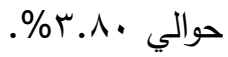
جدول رقم (؛ ): الوضع الحالي والمستقبلي لأهم المتغيرات الإتتاجية والاقتصادية المرتبطة بمحصول الياسمين

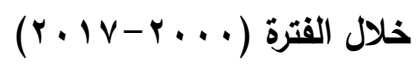

\begin{tabular}{|c|c|c|c|c|c|}
\hline \multirow[b]{2}{*}{ (مليونة جنيهة) } & \multicolumn{2}{|l|}{ السعر } & \multirow[b]{2}{*}{ (بالطنتاجية/فان) } & \multirow[b]{2}{*}{ (بالقدان) } & \multirow[b]{2}{*}{ السنوات } \\
\hline & جنيالأفرعي & كمية الإنتاج & & & \\
\hline YI. $\leqslant \varepsilon$ & r.1. & $741 . \wedge$. & r. $\varepsilon$ & Tr. & r... \\
\hline 19.9. & r. & $7) 9 . \wedge r$ & r.91 & rIT & $r \ldots l$ \\
\hline 11.71 & r... & 011.10 & $r .90$ & $19 V$ & $r \ldots r$ \\
\hline rI.VT & M.Y. & $971 . r$. & $\{., Y$ & rr. & $r \ldots r$ \\
\hline & T.Yo & $1.1 \leq .7$ & r.10 & rot & $r \ldots \varepsilon$ \\
\hline VT. 70 & Tr.vo & $r Y \leqslant \Lambda . T \leqslant$ & r.l & V1T & $r \ldots o$ \\
\hline 01.99 & r. זיז & $1 \leqslant \varepsilon \cdot .97$ & r.17 & $\{07$ & $r . . T$ \\
\hline $01 . \leqslant T$ & r. 99 & $1 \leqslant r T . \leqslant r$ & T.1ะ & sor & $r \ldots v$ \\
\hline Or.Y. & rV.Ar & $M \mu \cdot . r \leq$ & T.TE & \&YT & $r \ldots \lambda$ \\
\hline $0 V .01$ & r.. 0. & $1 \leqslant 07 . \ldots$ & r.r. & $\{00$ & $r \ldots q$ \\
\hline $0 \leqslant . \mu \Lambda$ & $\leqslant 1 . \leqslant \Lambda$ & $1 T 1 . .0 Y$ & T.YT & $\varepsilon \cdot r$ & $r \cdot 1$. \\
\hline 00.74 & $\varepsilon r .07$ & IrVO.9Y & T.YA & r人q & $r .11$ \\
\hline 79.70 & $\varepsilon 0 . V r$ & $10 r \cdot .9$. & r.ro & $\leqslant 0 \leqslant$ & $r \cdot I r$ \\
\hline ז... & $\varepsilon \Lambda . \cdot r$ & $|r| \cdot . \varepsilon r$ & r.71 & אדי & $r .1 T$ \\
\hline$\vee \wedge .71$ & $\leqslant \wedge .9 \wedge$ & $17 . \varepsilon . \varepsilon$ & $\varepsilon . r_{.}$ & rAT & $r \cdot 1 \varepsilon$ \\
\hline $9 V .0 V$ & $\leqslant 9.97$ & $190 \leqslant .7 \leqslant$ & $\varepsilon . Y \varepsilon$ & $\leq 71$ & $r .10$ \\
\hline $10 \leqslant . \leqslant 9$ & or. $\leqslant T$ & $r . r q . T \leq$ & 0.19 & 017 & $r .17$ \\
\hline 199.47 & 70.01 & $r \cdot \varepsilon l . \varepsilon r$ & $\varepsilon . \wedge r$ & וT & $r . I V$ \\
\hline $90 . \wedge 1$ & $\$ 1.1$ & $1 \leqslant 94.11$ & $r .01$ & $4.7 .7 \mathrm{~V}$ & المتوسط العام \\
\hline 111.71 & or... & $r 19 . . r$ & $\{.00$ & $\leq V \cdot .7$ & 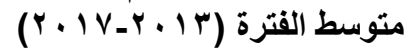 \\
\hline$\leq 7 . Y^{\circ}$ & 9.rV & $v \cdot v_{.} \cdot r$ & $\cdot \wedge$. & IrV.qY & الاتحراف المعياري \\
\hline \multicolumn{6}{|c|}{ تتبؤ الأريما } \\
\hline TrI.VT & $77 . \vee$. & TrYะ.17 & $\varepsilon . .9$. & TVA. \&. & $r \cdot r)$ \\
\hline$r \leqslant 1 . V q$ & $\uparrow \Lambda . \varepsilon$. & roro... & $0 .$. & $v \cdot v_{.}$. & $r \cdot r r$ \\
\hline$r V \cdot . V \cdot$ & $v \cdot .0$ & rᄉrq.7人 & $0 . r$. & $V \Gamma \Lambda . \varepsilon \cdot$ & $r \cdot r r$ \\
\hline$r 9 \Lambda . r \leqslant$ & VY.q. & $\varepsilon .91 . V$ & $0 . r$. & VVI.9. & $r \cdot r \varepsilon$ \\
\hline r ro.71 & vo.7. & $\varepsilon \varepsilon \varepsilon \cdot .10$ & 0.0 & A.v.r. & r. ro \\
\hline
\end{tabular}

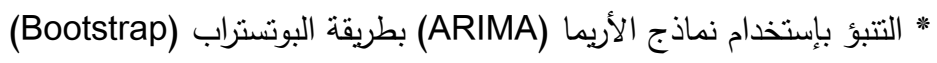
** تم حساب الإنتاجية بقسمة الإنتاج على المساحة لفترة التتبؤ المصدر:جمعت وحسبت من بيانات الجهاز المركزي للتعبئة العامة والإحصاء. Sources: http://www.fao.org/faostat/en/\#data 
جدول رقم (•): معادلات الإتجاه الزمني ونماذج الأريما لتطور المتغيرات الإنتاجية والاقتصادية لمحصول

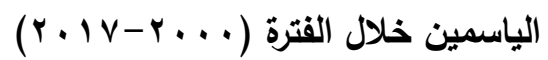

\begin{tabular}{|c|c|c|c|c|c|c|}
\hline No. & Variables & Equation* & $\mathbf{R}^{2}$ & $\mathbf{F}$ & Elasticity & $\begin{array}{l}\text { Marginal } \\
\text { Effect }\end{array}$ \\
\hline \multirow[t]{2}{*}{1} & 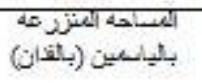 & $\begin{aligned} \operatorname{Ln} Y_{1} & =249.745+0.045 X \\
& (7.216) \quad(3.524)\end{aligned}$ & 0.44 & 12.42 & 3.83 & 32.56 \\
\hline & $\begin{array}{c}\text { ARIMA } \\
\log (1,0,1) \\
\text { RMSE }= \\
0.0974\end{array}$ & $\log Y_{1}=0.4304 \log A^{* 4}(1)-0.2713 \log M^{* 4 *(1)}$ & & & & \\
\hline \multirow[t]{2}{*}{2} & 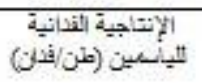 & $\begin{aligned} \operatorname{Ln} Y_{2}= & 2.723+0.027 X \\
& (14.498)(4.208)\end{aligned}$ & 0.53 & 17.69 & 0.30 & 2.55 \\
\hline & $\begin{array}{c}\text { ARIMA } \\
\log (1,0,1) \\
\operatorname{RMSE}= \\
0.0581\end{array}$ & $\begin{array}{l}\log Y_{2}=0.6234 \log A_{R}^{* 4}(1)+0.3513 \log \\
M^{* 4 *(1)}\end{array}$ & & & & \\
\hline \multirow[t]{2}{*}{3} & اليانسين (بالطعن) & $\begin{aligned} \operatorname{Ln} Y_{3}= & 679.990+0.072 X \\
& (7.208) \quad(5.611)\end{aligned}$ & 0.66 & 31.49 & -146.69 & -1246.87 \\
\hline & $\begin{array}{c}\text { ARIMA } \\
\text { Log }(1,0,0) \\
\text { RMSE }= \\
0.104\end{array}$ & $\log Y_{3}=0.4860 \log A R^{88}(1)$ & & & & \\
\hline \multirow[t]{2}{*}{4} & 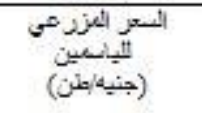 & $\begin{aligned} \operatorname{Ln} \mathrm{Y}_{4}= & 28.007+0.038 \mathrm{X} \\
& (34.403)(14.174)\end{aligned}$ & 0.93 & 200.91 & -10.05 & -85.45 \\
\hline & $\begin{array}{c}\text { ARIMA } \\
\log (1,0,1) \\
\operatorname{RMSE}= \\
0.0228\end{array}$ & $\begin{array}{l}\log Y_{d}=0.5711 \log A^{* 4}(1)+0.8336 \log \\
\operatorname{MA}^{* 4+(1)}\end{array}$ & & & & \\
\hline \multirow[t]{2}{*}{5} & 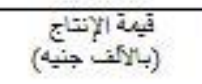 & $\begin{aligned} \operatorname{Ln} Y_{5}= & 19.109+0.109 \times \mathrm{XT} \\
& (7.474) \quad(8.857)\end{aligned}$ & 0.83 & 78.45 & 0.97 & 0.08 \\
\hline & $\begin{array}{c}\text { ARIMA } \\
\log (1,0,0) \\
\text { RMSE = } \\
0.1000\end{array}$ & $\log Y_{5}=0.5543 \log A R^{* 8}(1)$ & & & & \\
\hline
\end{tabular}

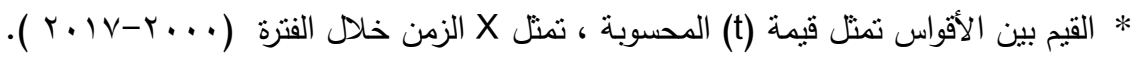
Auto Regressive (AR)*** الإنحدار المتسلسل. Mouving Average (MR)*** المصدر: جمعت وحسبت من نتائج تحليل الإتجاه الزمني ونماذج الأريما لبيانات الجدول رقم (ع).

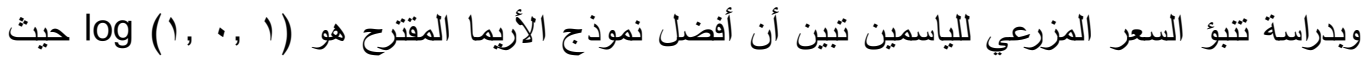

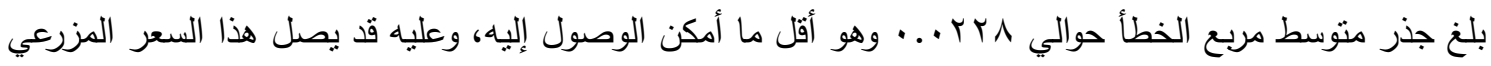

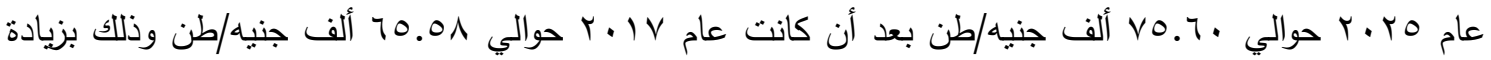

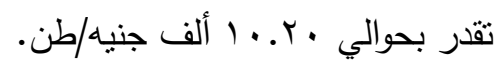
- قيمة إنتاج الياسمين: - n

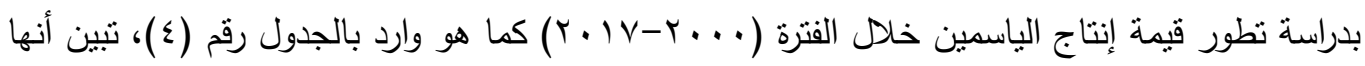

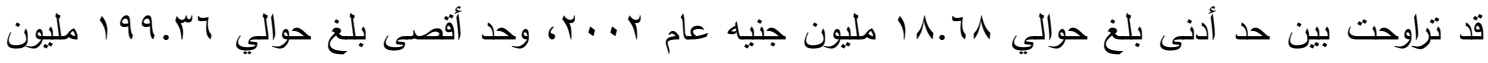




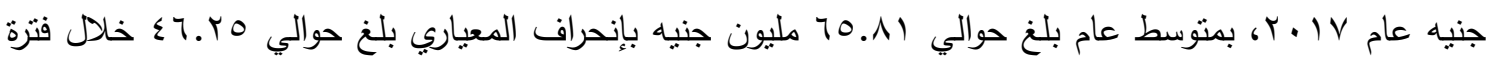

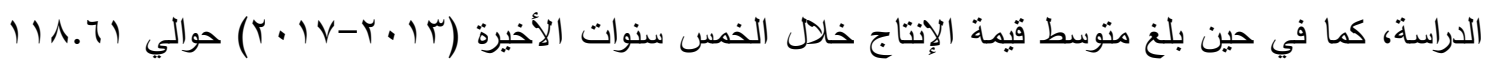

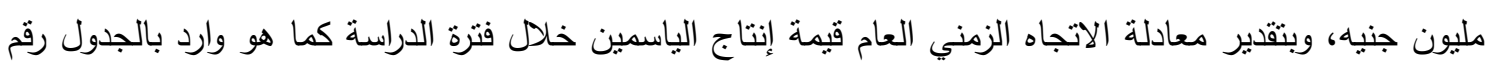

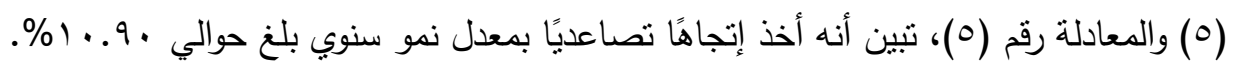

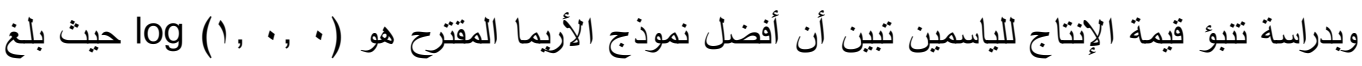

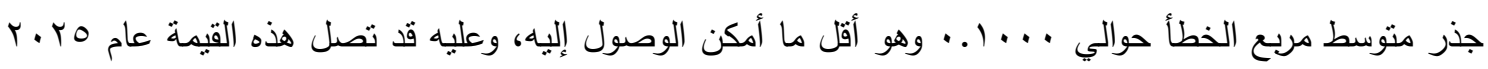

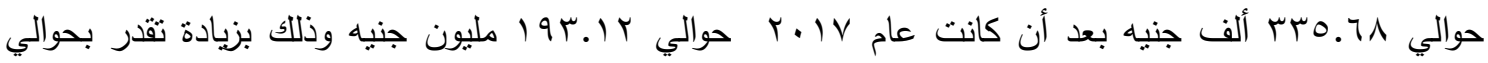

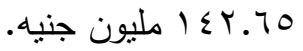

ه. دراسة للوضع التصديري للنباتات الطبية والعطرية ومنتجات الياسمين: ه - 1 واردات وصادرات النباتات الطبية والعطرية: بدراسة تطور واردات وصادرات النباتات الطبية والعطرية يتبين الأتي: وادراتي

- كمية واردات النباتات الطبية والعطرية:

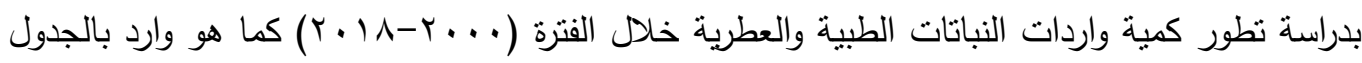

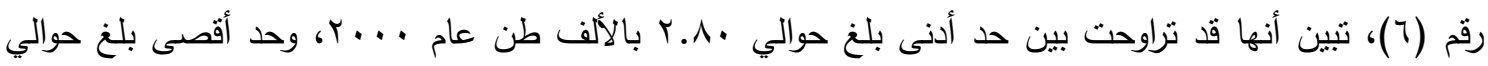

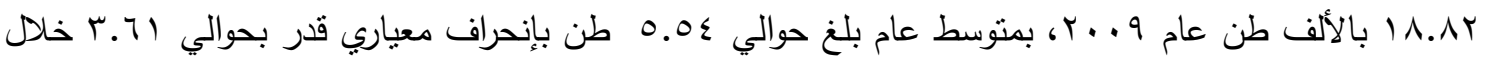

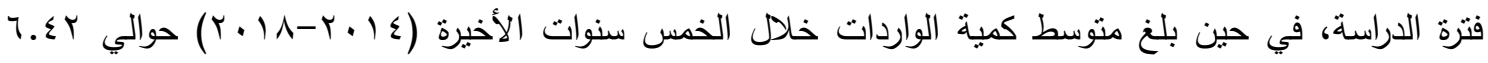

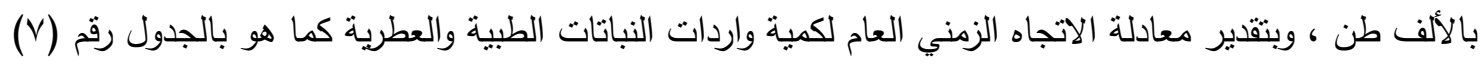

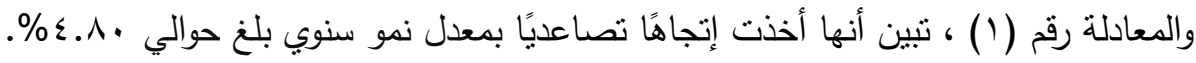

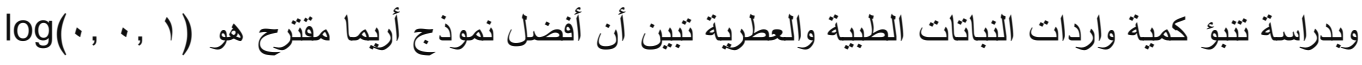

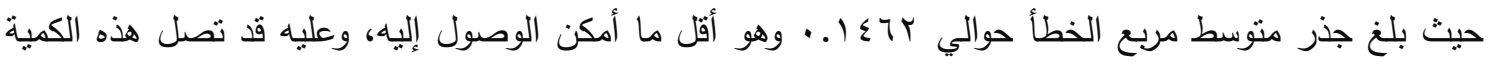

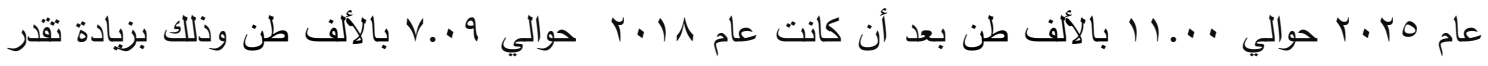
بحوالي 19. ب بالألف طن.

- قيمة واردات النباتات الطبية والعطرية:

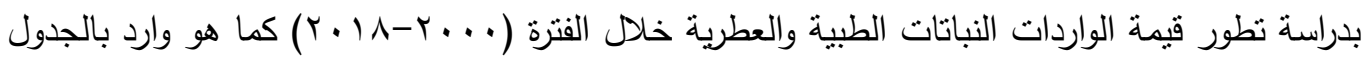

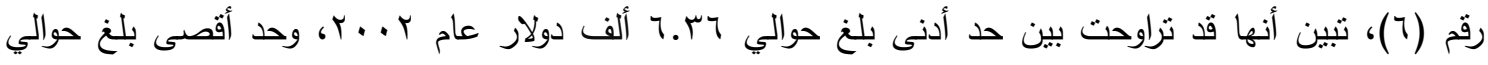

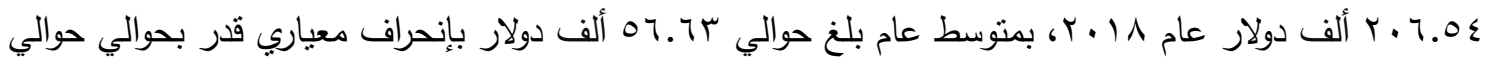

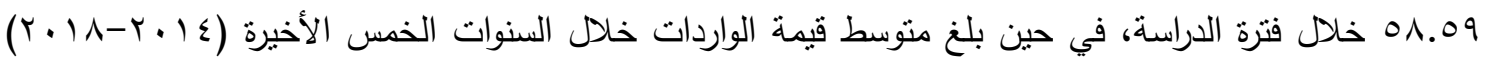
حوالي 11 V.00 ألف دولار، وبتقدير معادلة الاتجاه الزمني العام لقيمة واردات النباتات الطبية خلال تللك الفترة

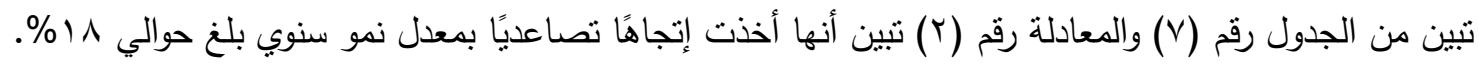

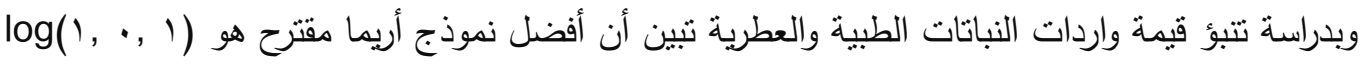

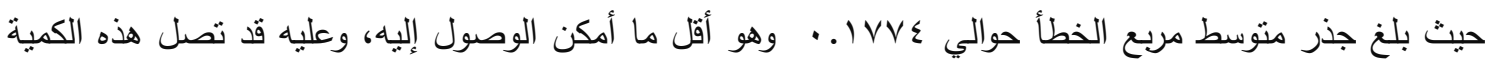




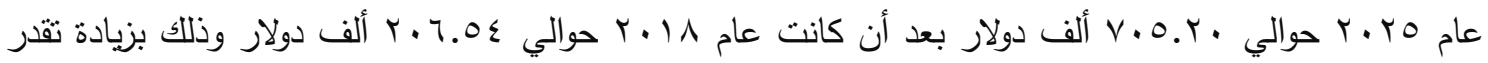
بنحو

- كمية صادرات النباتات الطبية والعطرية:

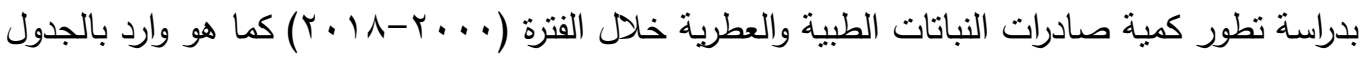

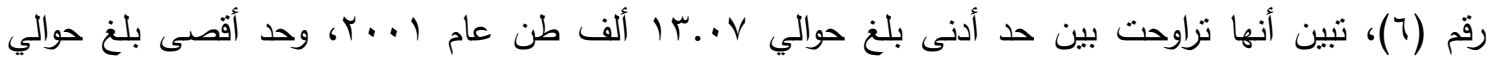

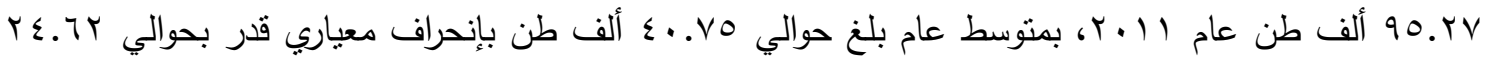

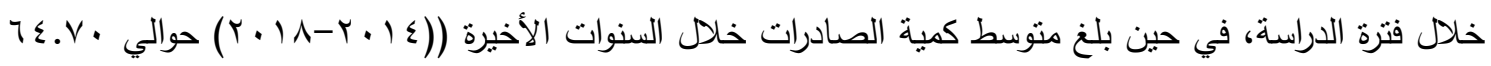
ألف طن، وبتقدير معادلة الاتجاه الزمني العام لكمية صادرات النباتات الطبية خلال تلك الفترة تنبين من الجدول

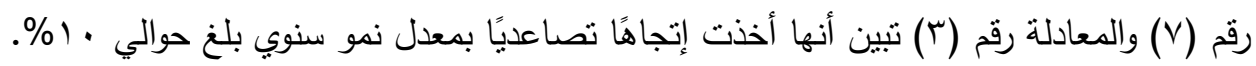

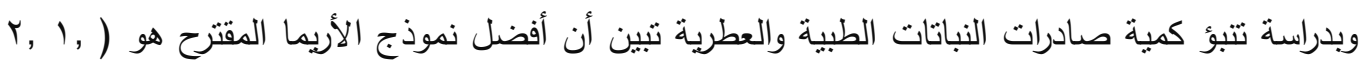

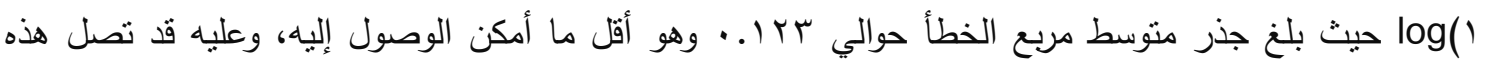

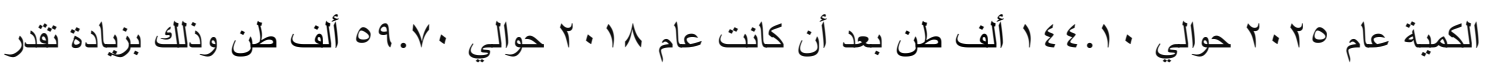

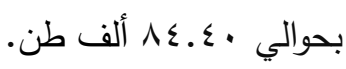

- قيمة صادرات النباتات الطبية والعطرية:

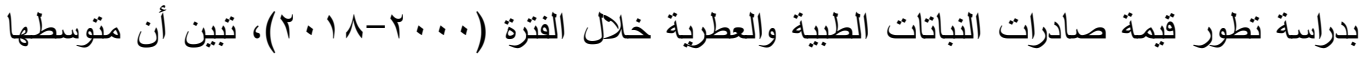

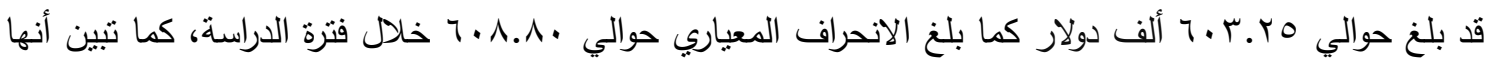

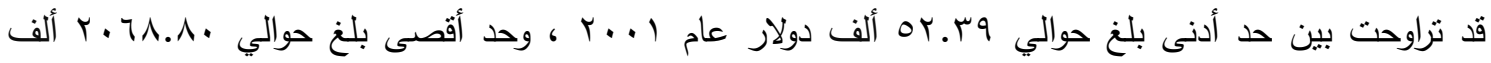

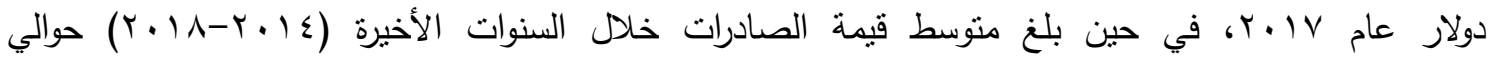

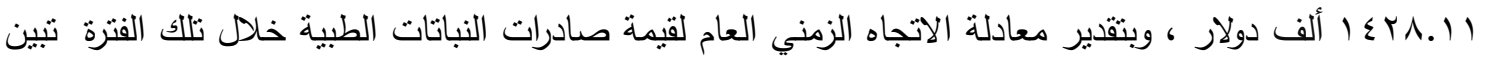

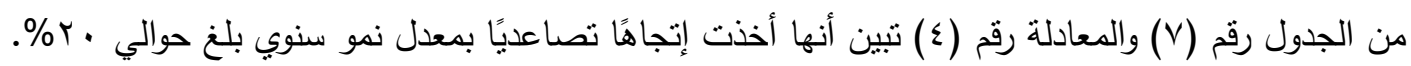
وبدراسة تتبؤ قيمة صادرات النباتات الطبية والعطرية تبين أن أفضل نموذج الأريما المقترح هو ( , . , 1.

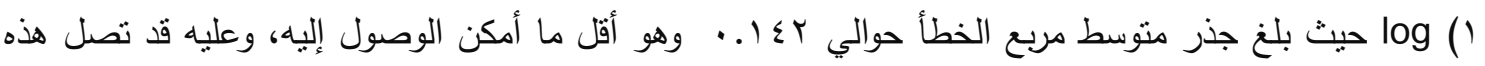

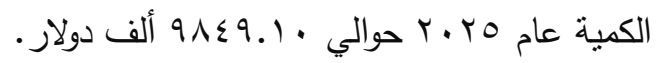




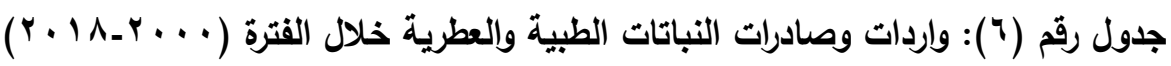

\begin{tabular}{|c|c|c|c|c|}
\hline \multicolumn{2}{|c|}{ الصادرات } & \multicolumn{2}{|c|}{ الواردات } & \multirow{3}{*}{ السنوات } \\
\hline القيمة & الكمية & القيمة & الكمية & \\
\hline (بالألف دولار) & (بالألف الطن) & (بالألف دولار) & (بالألف الطن) & \\
\hline 70.91 & $1 V . \leqslant 9$ & $\Lambda . K^{\prime}$ & r.A. & $r \ldots$ \\
\hline or.rq & $1 r . v$ & $\Lambda . r T$ & r.v. & $r \ldots l$ \\
\hline 79.10 & 17.81 & 7.47 & חז.ז & $r \ldots r$ \\
\hline ITO.rT & $17 . V r$ & 11.21 & r.ro & $r \ldots r$ \\
\hline $10 \% .09$ & 11.00 & $1 \leq .9 r$ & $\varepsilon .+1$ & $r \ldots \varepsilon$ \\
\hline $1 \leqslant V .9 V$ & $11 . \leqslant 9$ & 11.19 & r.10 & $r \ldots o$ \\
\hline IVT.E. & $Y 1.01$ & $1 \pi . \leqslant 7$ & $\varepsilon . \Upsilon \wedge$ & $r \ldots r$ \\
\hline 111. Or & YY.OV & 19.01 & $\varepsilon .9 V$ & $r \ldots V$ \\
\hline$r 0 . . \leqslant 0$ & T!.ᄉI & rq..$<\varepsilon$ & r. ro & $r \cdots \wedge$ \\
\hline$\{00.00$ & rᄉ. 79 & 121.11 & $1 \Lambda . \wedge r$ & $r \ldots q$ \\
\hline 011.71 & «). . & r^.71 & r. 19 & $r \cdot 1$. \\
\hline$V \varepsilon \cdot . \varepsilon 1$ & $90 . Y V$ & $\{0.91$ & $0.0 Y$ & $r+11$ \\
\hline OYะ.17 & rᄉ.r. & Or.rV & $7 . \Gamma \varepsilon$ & $r .1 T$ \\
\hline$V 7 . .1 \varepsilon$ & $7 . .01$ & $T V . \cdot \varepsilon$ & $V . \cdot V$ & $r .14$ \\
\hline$q \leqslant r . r$ & $\vee \wedge .79$ & 70.19 & 0.10 & $r \cdot 1 \varepsilon$ \\
\hline $9 Y 7.41$ & $00.1 \wedge$ & Tr. & $1 . .0$ & $r .10$ \\
\hline זיד.ואו & $V Y . \leqslant \Lambda$ & $9 \cdot . \leqslant 1$ & $7 . \leqslant 9$ & $r .17$ \\
\hline 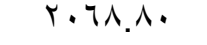 & 07.17 & $1 \pi 1 . \leqslant 9$ & $\varepsilon . \vee 1$ & r. IV \\
\hline $191 \cdot . \wedge r$ & 09.7. & $r .7 .0 \varepsilon$ & $V .99$ & $r .11$ \\
\hline T.Y.YO & $\varepsilon \cdot . \vee 0$ & 07.74 & 0.04 & المتوسط العام \\
\hline $1 \leq r \wedge .11$ & $T \leqslant . V$. & 118.00 & $T . \leqslant Y$ & 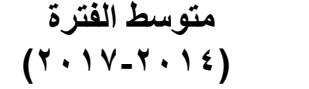 \\
\hline \multirow[t]{2}{*}{$r .1 . r Y$} & $r \varepsilon .7 Y$ & $0 \wedge .09$ & 5.71 & الانحراف المعياري \\
\hline & & تتبؤ الأريما & & \\
\hline$\sum r \cdot 7.0$. & 97.0. & $r \leqslant r . \wedge$. & 9.1. & $r \cdot r)$ \\
\hline$r \leqslant r \cdot . r$. & $\left.1 \cdot r_{.}\right)$ & $\varepsilon 1 \cdot . \wedge$. & 9.0. & $r \cdot r{ }^{2}$ \\
\hline $7 \leq 09.1$. & $|r| . \wedge$. & $\leqslant 9 r_{.}$. & $1 \cdot \ldots$ & $r \cdot r r$ \\
\hline$q \pi \leq 1 .$. & 1ro. 9. & $0 \wedge 9.1$. & $1 \cdot . \varepsilon$ & $r \cdot r \varepsilon$ \\
\hline $9 \wedge \leq 9.1$. & $1 \leq \varepsilon .1$. & V.o.r. & $11 .$. & T. YO \\
\hline
\end{tabular}

Sources: http://www.fao.org/faostat/en/\#data 
جدول رقم (V): معادلات الإتجاه الزمني ونماذج الأريما لتظور المتغيرات الإنتاجية والاقتصادية للنباتات الطبية

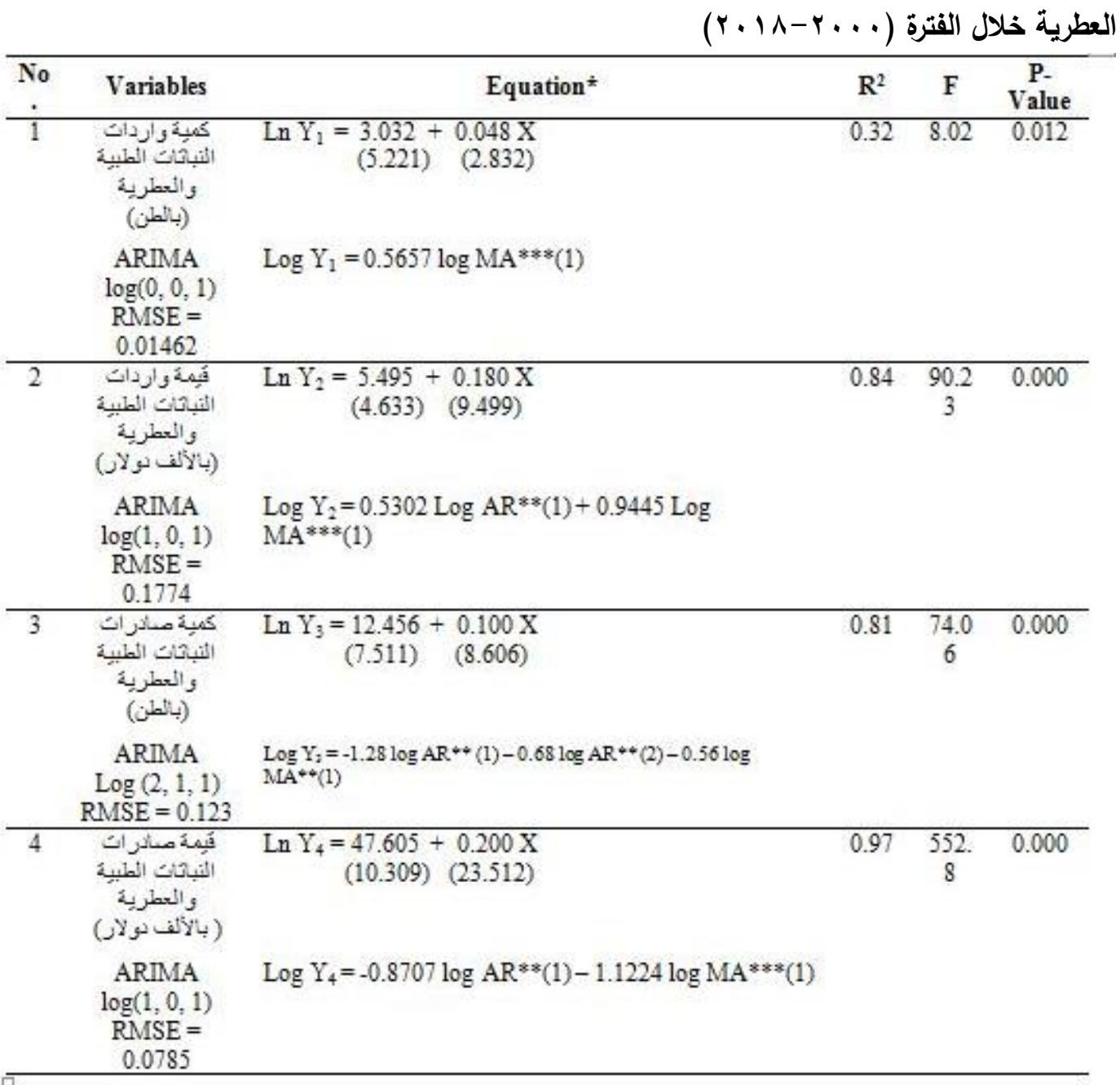

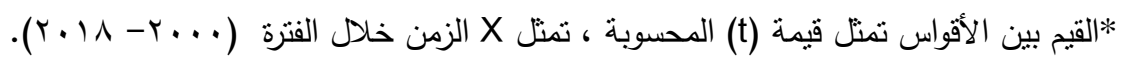
Auto Regressive (AR)** الإنحدار المتسلسل. Mouving Average (MR)*** المنوسط المتحرك.

المصدر: حسبت من بيانات الجدول رقم (V) باستخدام البرنامج الإحصائي Spss. P- الوضع التصديري لمنتجات الياسمين: بدراسة نطور تطور الوضع التصديري لمنتجات الياسمين (عجينة ، زيت) يتبين الأتي: - كمية صادرات عجينة الياسمين:

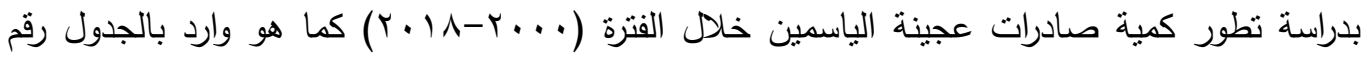

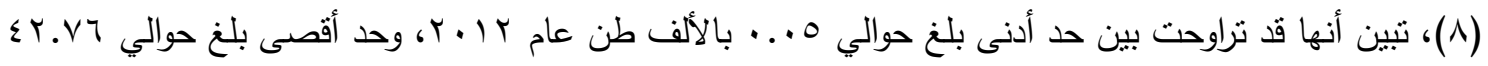

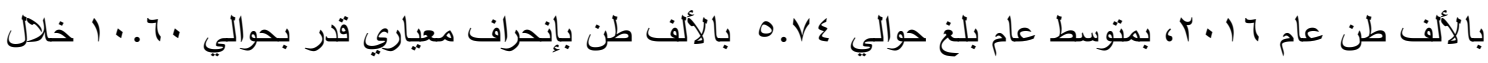

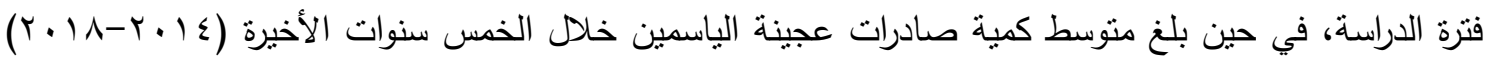
حوالي 91 ـ 17 بالألف طن، وبتقدير معادلة الاتجاه الزمني العام لكمية صادرات عجينة الياسمين خلال تلك الفترة كما هو وارد بالجدول رقم (9) والمعادلة رقم (1) تبين عدم الاستقرار بين الزيادة والنقصان وقد بلغ مقدار التغير

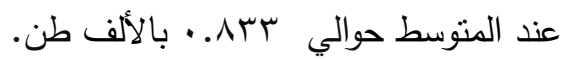


- قيمة صادرات عجينة الياسمين:

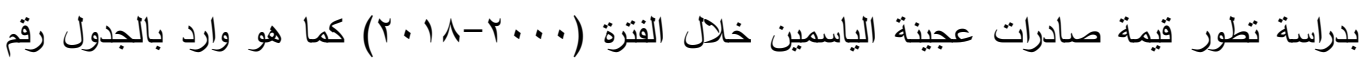

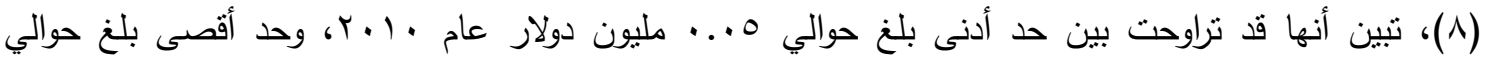

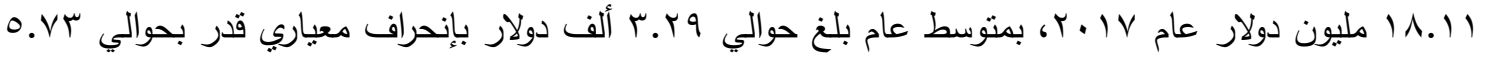

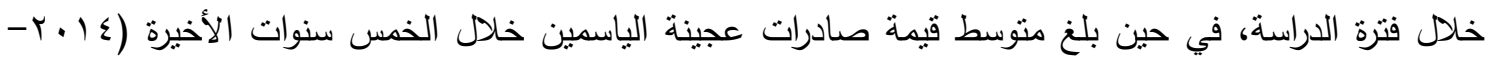

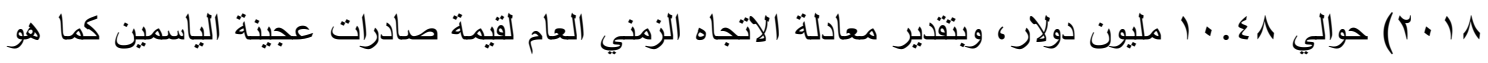

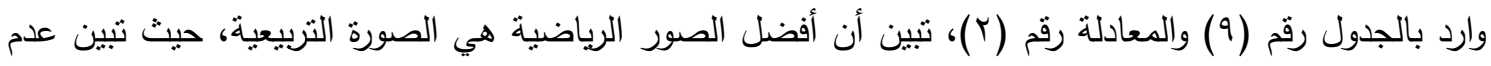

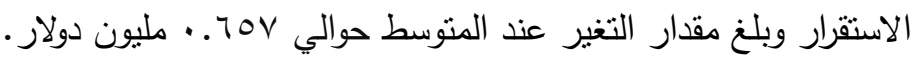
- كمية وقيمة صادرات زيت الياسمين:

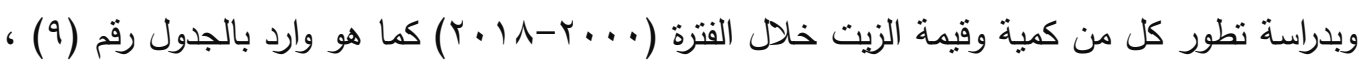
تبين عدم وجود البيانات الكافية والوافية عن صادرات زيت الباسمين العطري من قبل الجهاز المركزي للتعبئة العامة

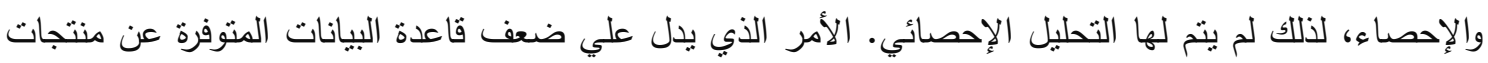
محصول الياسمين وبصفة خاصة زيت الياسمين.

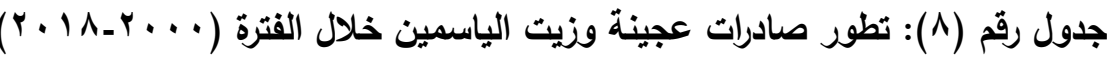

\begin{tabular}{|c|c|c|c|c|}
\hline \multicolumn{2}{|c|}{ زيت الياسمين } & \multicolumn{2}{|c|}{ عجينة الياسمين } & \multirow[b]{2}{*}{ السنوات } \\
\hline القيمة (بالألف دولار) & الكمية (بالكجم) & $\begin{array}{c}\text { القيمة (بالمليون } \\
\text { دولار) }\end{array}$ & الكمية (بالف & \\
\hline Trद.1. & rqV & I. & 0.11 & r... \\
\hline או TMT & $1 \leqslant 7$. & $1 . \leqslant 9$ & r. 19 & $r \ldots l$ \\
\hline$r \leqslant 7.9 T$ & 11.99 &. .70 & $\varepsilon .0 r$ & $r \ldots r$ \\
\hline r Tq.rT & 1701 & $\cdot .9 \leqslant$ & $\varepsilon . \varepsilon V$ & $r \ldots r$ \\
\hline IVT.VO & $r q$. & • & 1.19 & $r \ldots \varepsilon$ \\
\hline 77.91 & orl & .0 . & $\cdot .71$ & $r \ldots o$ \\
\hline$\S 1.1 \%$ & 77. &. . &.$V \varepsilon$ & $r \ldots r$ \\
\hline 1.91 & v &.$\mu$. & ד4 & $r \ldots v$ \\
\hline - & - & $.0 \%$ &.$\vee \vee 9$ & $r \ldots \Lambda$ \\
\hline - & - & .00 & $0 . \leqslant 0$ & $r \ldots q$ \\
\hline - & - &. .0 & $1.1 \varepsilon$ & $r \cdot 1$. \\
\hline - & - & $r . .$. & $\cdot . \varepsilon \varepsilon$ & $r .11$ \\
\hline - & - & $\because \wedge$ &. .0 & $r .1 r$ \\
\hline 199.99 & TVT &..$\leqslant r$ & - ro & $r .1 T$ \\
\hline $1.1 . r_{0}$ & $\varepsilon \varepsilon$ & r.vq & $\varepsilon . V V$ & $r \cdot 1 \leq$ \\
\hline $9 \leqslant . \leqslant \varepsilon$ & $\leq \pi r$. & 5.17 & .91 & $r .10$ \\
\hline - & - & 11.9. & $\varepsilon Y . V T$ & $r .17$ \\
\hline 1.1 .00 & 187. & 11.11 & $V, Y V$ & $r . I V$ \\
\hline - & - & $1 V . \varepsilon \varepsilon$ & ro.17 & $r \cdot 11$ \\
\hline - & - & $r . r q$ & $0 . V \leqslant$ & المتوسط العام \\
\hline - & - & $1 \cdot . \leqslant 1$ & 17.19 & متوسط الفترة \\
\hline - & - & $0 . \wedge \wedge$ & $1 . .7$ & الأنحر اف المعياري \\
\hline
\end{tabular}

Sources: http://www.fao.org/faostat/en/\#data 


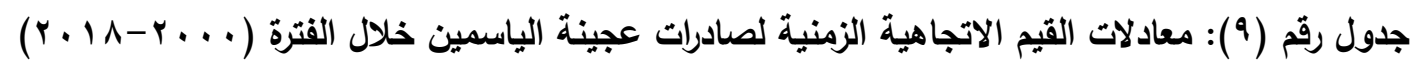

\begin{tabular}{|c|c|c|c|c|c|}
\hline No. & Variables & Equation* & $\mathrm{R}^{2}$ & F & Sig. \\
\hline 1 & 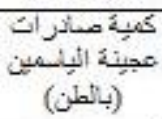 & $\mathrm{Y}_{1}={ }_{(1.598)} 10.419-2.887 \mathrm{X}+0.186 \mathrm{X}^{2}$ & 0.43 & 6.02 & 0.011 \\
\hline 2 & 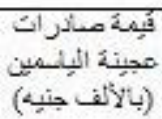 & 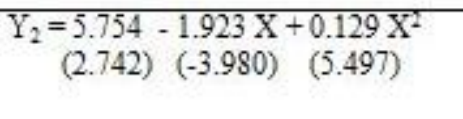 & 0.80 & 31.57 & 0.000 \\
\hline
\end{tabular}

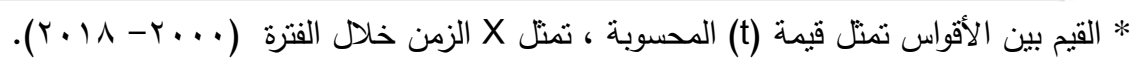

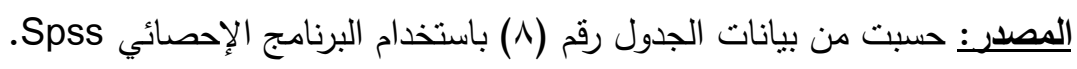
ـ التسويق الداخلي لمحصول الياسمين:

تنين من الدراسة الميدانية في منطقة شبرا بلولة بمحافظة الغربية إمكانية وضع تصور للمسلك التسويقي

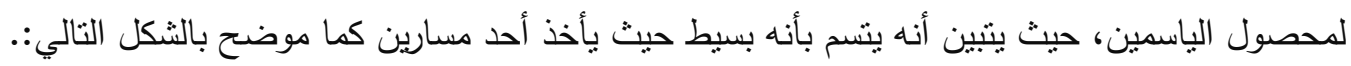

المسار الأول: يقوم المزارع بجمع محصوله من أزهار الياسمين ونقله مباشرة للمصنع ويتم عملية البيع والثراء. المسار الثاني: يقوم المزارع بجمع المحصول من أزهار الياسمين وبيعه إلى الوسيط (المجمع) ثم يقوم المجمع بنقل وبيع المحصول للمصنع مباشرة. الثكل التصوري للمسلك التسويقي الداخلى لمحصول الياسمين

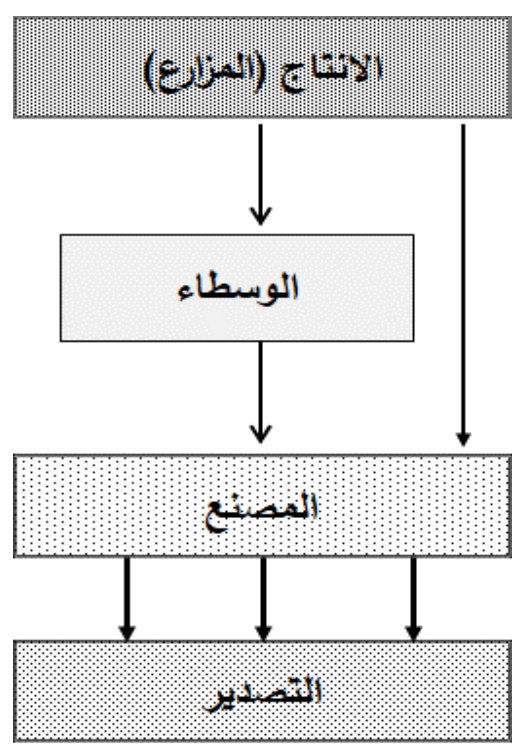

المصدر: بيانات الدراسة الميدانية بمنطقة شبرا بلولة بمحافظة الغربية خلال العام الزراعي (1/ • ب/9 • ؟).

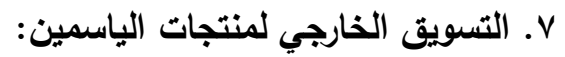
يعد النهوض بقطاع الصادرات المصرية هو العامل الرئيسي لعملية النتمية الإقتصادية، وذلك من عن طريق دعم قطاع التصدير الزراعي منمثلة في صادرات النباتات الطبية والعطرية بصفة عامة والياسمين بصفة

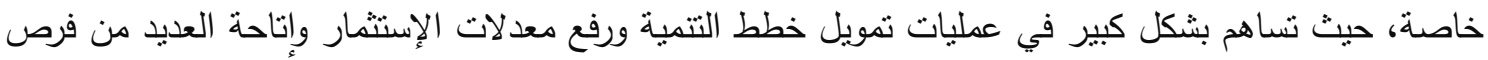

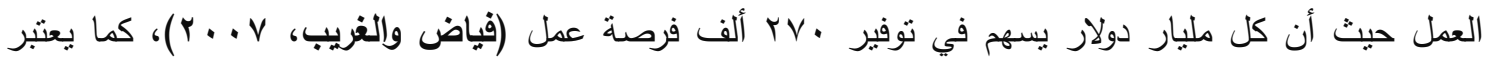
محصول الياسمين من المحاصيل التصديرية الغير تقلبدية الهامة والمتميزة ذات الجودة العالية، وتبين من الجدول رقم (• () أن الدول المستورة لمنتجات الياسمين المصري معظمها دول أوربية بالاضافة إلي الولايات المتحدة دئهين 


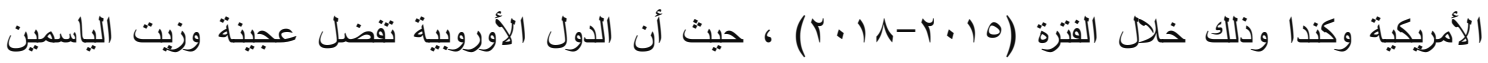
المصري عن منافسه الهندي، لذا يجب على الدولة الإهتمام بالنباتات الطبية والعطرية وخاصة محصول الياسمين

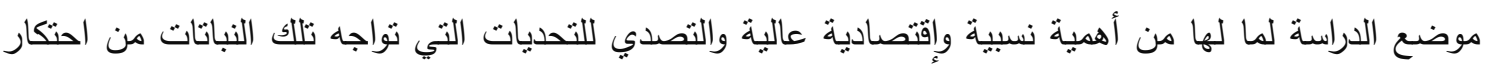
وعدم التوسع في المساحة الزراعية لها.

جدول رقم ( • (): إنتاج أهم الدول التي تستورد من مصر منتجات الياسمين بالكجم خلال الفترة (0 ـ ب $(r \cdot 1 \Lambda$

\begin{tabular}{|c|c|c|c|c|}
\hline \multicolumn{4}{|c|}{ السنوات } & \multirow[b]{2}{*}{ الدولة الدولة } \\
\hline$r \cdot 1 \Lambda$ & $r \cdot I V$ & $r .17$ & $r .10$ & \\
\hline 1.9 .5 & r^.० & $r \leqslant 0$ & Tr.t & فرنسا \\
\hline iv & · & 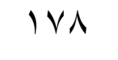 & rA. 0 & الو لايات المتحدة الأمريكية \\
\hline . & - & - & r & المملكة المتحدة \\
\hline · & · & - & rr & سويسر ا \\
\hline $19 . \varepsilon$ & · & · & · & هنغاريا \\
\hline $10 . r$ & · & · & · & أير لندا \\
\hline - & - & $1 \leqslant$ & - & هولندا \\
\hline 9.V & (T.r & · & · & 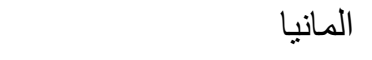 \\
\hline$\varepsilon .1$ & - & • & - & كندا ا \\
\hline
\end{tabular}

\section{Sources: http://www.expoegypt.gov.eg}

توصي الدراسة في ضوء النتائج المتحصل عليها بما يلي: (1) توفير البيانات عن الأسواق الداخلية والخارجية والخدمات التسويقية للياسمين من نقل وتخزين وتسويق وغيرها، (r) التغلب على المعوقات التى تواجه محصول الياسمين سواء أثثاء الانتاج والتسويق، (ب) العمل على تدعيم تتافسية منتجات الياسمين السوق الخارجى، (r) العمل تصنيع منتجات نهائية من عجينة الياسمين والزيت والاستفادة من القيمة المضافة بدلا من تصديرهما خام، (ع) توفير مصادر تمويل مناسبة من حيث طريقة التعامل وسعر الفائدة والوقت الذي ينتاسب مع اللمنتجين الحاليين والمرتقبين. (0) تشجيع تكوين تعاونيات إنتاجية وتسويقية مرنبطة بمزارعي الياسمين علي مستوي

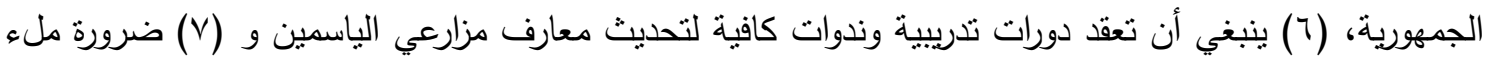
الفجوة الناجمة عن سوء الاتصال مع المرشدين في هذا المجال. 


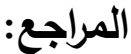

الجهاز المركزى للتعبئة العامة والإحصاء، النشرة السنوية لإحصاءات المساحات المحصولية والإنتاج النباتى ،

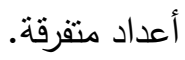

الجهاز المركزى للتعبئة العامة والإحصاء ، نثرة التجارة الخارجية، أعداد متفرقة.

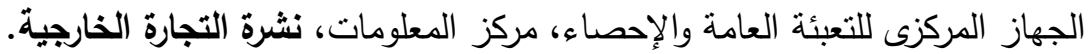

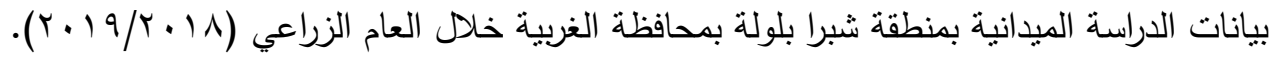

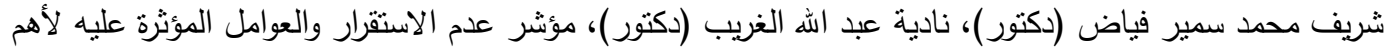

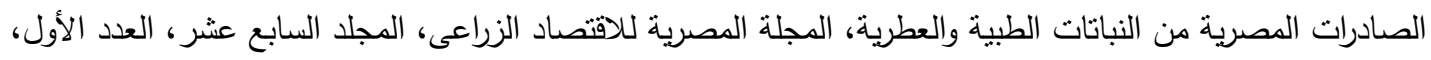

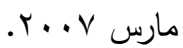




\title{
SUMMARY
}

\section{Economics of Production and Marketing of the Egyptian Jasmine Crop}

\author{
Mohamed I. M. Elshahawy, Dina M. A. Elshaer, Jacqueline A. Jabra \\ Faculty of Agriculture, Saba Pasha-Alexandria University
}

\begin{abstract}
The research is aimed to study the economics of production and marketing of the Egyptian jasmine crop during the period (2000-2017). This goal can be achieved through the following objectives: (1) Study the relative importance of both medicinal, aromatic plants and jasmine in relation to the cultivated area, (2) Study the development of some economic variables Important issues related to the economics of production and marketing of medicinal, aromatic plants, the harvest of jasmine and their future forecasting, (3) An assessment of the export situation of medicinal, aromatic plants and jasmine products and (4) A set of recommendations that could benefit economic policy makers in this field.
\end{abstract}

The results of the research revealed the following: (1) The relative importance of the jasmine crop represents about $0.57 \%$ of the total area of medicinal and aromatic plants, and the area cultivated with jasmine during the period (2000-2017) took an upward trend with an annual growth rate of about $4.50 \%$, (2) Average farm price of jasmine during the study period was about 41.10 thousand pounds / ton with a standard deviation estimated at 9.27, and it was found that it took an upward trend with an annual growth rate of about $3.80 \%$, and therefore this farm price in 2025 may reach about 75.60 thousand pounds/ton, after it was about 65.58 thousand pounds/ton in 2017, with an increase of about 10.20 thousand pounds/ton, (3) The average value of jasmine production during the study period was 65.81 million pounds, with a standard deviation of about 46.25 , and it was found that it took an upward trend during that period with an annual growth rate of about $10.90 \%$, and therefore this value may reach in 2025 to 335.68 thousand pounds after That the year 2017 was about 193.12 million pounds, an increase of 142.65 million pounds, (4) The average quantity of jasmine paste exports during the study period was about 5.74 tons, with a standard deviation estimated at 10.60, and it was found to be unstable between increase and decrease, and the average amount of change was about 0.833 tons, (5) The average value of jasmine paste exports during the study period was $\$ 3.29$ million, with a standard deviation estimated at 5.73 . It was also found to be unstable between increase and decrease, and the average amount of change was $\$ 0.657$ thousand, (6) The study revealed the lack of sufficient and adequate data from exports of jasmine essential oil, which indicates the weakness of the available database on jasmine products, especially jasmine oil, (7) The marketing approach of the jasmine crop in internal marketing takes two paths: the first path: the farmer collects his crop of jasmine flowers and transfer it directly to the factory, and the buying and selling process is completed, the second track: the farmer collects the crop from the jasmine flowers and sells it to the mediator (the collector), then he The collector transfers and sells the crop directly to the plant and (8) The jasmine crop is considered one of the important and distinguished non-traditional export crops of high quality, and it was found that the imported countries for the Egyptian jasmine products are mostly European countries in addition to the United States of America and Canada during the period (2015-2018), as European countries prefer Egyptian jasmine paste and oil About the Indian competitor.

The study recommends the following: (1) Providing data on internal and external markets and marketing services for jasmine, including transportation, storage, marketing, and others, (2) Overcoming the obstacles facing the jasmine crop both during production and marketing, (3) Working to strengthen the competitiveness of jasmine products on the external market, (4) Work to manufacture finished products from jasmine paste and oil and to benefit from the added value instead of exporting them raw, (5) Providing suitable sources of financing in terms of the method of dealing, the interest rate and the time that are commensurate with the current and potential producers, (6) Encouraging the formation of production and marketing cooperatives linked to jasmine farmers throughout the Republic, (7) Adequate training courses and seminars should be held to update the knowledge of jasmine growers and (8) The need to fill the gap caused by poor communication with mentors in this field.

Key words: production, marketing, exports, revenue, marketing behavior, forecasting, jasmine, medicinal and aromatic plants. 
